\title{
Some Contributions to the Theory of Transformation Monoids
}

\author{
A. Ballester-Bolinches* \\ E. Cosme-Llópez* \\ P. Jiménez-Seral ${ }^{\dagger}$
}

\begin{abstract}
The aim of this paper is to present some contributions to the theory of finite transformation monoids. The dominating influence that permutation groups have on transformation monoids is used to describe and characterise transitive transformation monoids and primitive transitive transformation monoids. We develop a theory that not only includes the analogs of several important theorems of the classical theory of permutation groups but also contains substantial information about the algebraic structure of the transformation monoids. Open questions naturally arising from the substantial paper of Steinberg [A theory of transformation monoids: combinatorics and representation theory. Electron. J. Combin. 17 (2010), no. 1, Research Paper 164, 56 pp] have been answered. Our results can also be considered as a further development in the hunt for a solution of the Černý conjecture.
\end{abstract}

Mathematics Subject Classification (2010): 16W22, 20M30

Keywords: monoid theory, monoid actions, transitive, faithful, primitive.

\section{Introduction}

The principal objects of interest in the present paper are finite nonempty sets and finite monoids acting on them. An action or act of a monoid $M$ on a nonempty set $\Omega$ is a monoid homomorphism from $M$ to the transformation monoid $\mathrm{T}_{\Omega}$ of $\Omega$. The action is faithful if the corresponding morphism is injective. In such case, we say that the pair $(\Omega, M)$ is a transformation monoid.

Actions are quite natural, and turns out to be a useful tool to study monoids. From an algebraic point of view, monoid actions can be seen as a generalisation of group actions in group theory. From the computer science perspective, monoid actions are closely related to automata; the set models the state space of the automaton and the action models transformations of states in response to inputs.

Transformation monoids play to some extent the role that is taken by permutation groups in group theory. One of these relations is manifested by the Schützenberger group of an element of a transformation monoid, which is represented by a permutation group rather than a group of transformations. Another relation which is used by most functions that deal with transformation monoids is the fact that a transformation monoid can be efficiently described in terms of several permutation groups ([17, 18]).

\footnotetext{
${ }^{*}$ Departament de Matemàtiques, Universitat de València; Dr. Moliner, 50; E-46100 Burjassot (València), Spain, email: Adolfo.Ballester@uv.es, Enric.Cosme@uv.es

${ }^{\dagger}$ Edificio de Matemáticas, Universidad de Zaragoza; Pedro Cerbuna, 12; E-50009 Zaragoza, Spain, email: paz@unizar.es
} 
The importance of permutation groups in the study of groups comes from a theorem of Cayley asserting that any group is isomorphic to a permutation group. The proof extends to monoids as well: every monoid is isomorphic to a transformation monoid. This classical result justifies the importance of these structures in semigroup theory and motivates their study as general algebraic objects.

Recent applications to decision problems, automata theory and Markov chains, for example, have strengthened the status and interest of the theory of transformation monoids. In fact, most of the books and research articles dealing with these structures focus on specific transformation monoids, and on computational, combinatorial or categorical issues (see for instance [6, 11, 14, 16]). The Cerný conjecture, for example, is an interesting problem in automata theory that naturally requires the study of full transformation monoids. Given a set of tranformations on $n$ letters such that some products of elements are a constant map, the Cerný conjecture states the existence of a product of length at most $(n-1)^{2}$ which is a constant map. There exists a vast number of papers on the subject $([26,19,20,24])$.

The research presented in this paper has its origin in an earlier work of Steinberg [25, where the ideas that have been used by several authors while working on the Černý conjecture are systematised and developed. The end result starts a theory of transformation monoids based on Green's theory [13] of induction and restriction functors relating an algebra $A$ with local algebras of the form $e A e$ with $e$ idempotent, and the theory of semigroup representations introduced by Clifford, Munn and Ponizovsky [6, 1]. The functorial approach used by Steinberg allows him to generalise results from modules to transformation monoids. It is based in the description of restriction and induction functors that provides a way of transforming monoid actions into group actions and vice versa.

The second part of Steinberg's paper is a first step in the program of understanding primitive transformation monoids. Steinberg obtains a generalization for transformation monoids of the classical result of D. Higman [8, 5] that states that a permutation group is primitive if and only if each associated orbital digraph is connected.

The underlying motivation of the present paper is to take the studies of transformation monoids as algebraic objects further. We exploit the dominating influence that permutation groups have on transformation monoids and we offer a complete description of transitive transformation monoids and primitive transformation monoids. We develop a theory that includes the analogs of several important theorems of the classical theory of permutation groups. These results answer some open questions naturally arising in the paper [25] and can be considered as further developments in the hunt for a solution of the Černý conjecture.

Studying transformation monoids by a complete analogy with permutation groups is not a good enterprise. Instead, one should benefit from the distinguishing feature of semigroups: the existence of proper ideals. Let us recall that transitive representations of finite monoids by total mappings correspond to the action by right multiplication on $e M$, with $e$ an idempotent in the minimal ideal of $M$. This is a particular case of the results proved in [21], where it is shown that transitive representations of finite monoids by partial mappings are precisely the quotients of Schützenberger representations by a right congruence.

In fact, the existence of a unique minimal ideal $\mathrm{I}(M)$ in a given finite monoid $M$ is a keystone in our work. The structure of the principal right ideal $e M$ characterises any monoid $M$ having a transitive and faithful action as a submonoid of a wreath product of a group with a right letter mapping monoid [2, 6, 7]. Moreover, the minimal ideal is a simple semigroup and it is, therefore, a disjoint union of isomorphic groups. Simple semigroups were completely determined up to isomorphism by Rees's theorem [22, 23] as Rees matrix semigroups. 
The groups $G_{e}$ included in the minimal ideal play an important role in the present work as transitive transformation monoids actually restrict to transitive permutation groups and we can establish a description based on the cosets of a core-free subgroup. For every core-free subgroup we set as many equivalence relations on the set of idempotents of $e M$ as the index of such subgroup in the group $G_{e}$. These equivalence relations, together with the study of the permutation representations of $G_{e}$ are crucial in our first main results: the description of all the congruences on $e M$ that lead to transitive transformation monoids (Theorems 17 and 20). These theorems can be used to construct all transitive and faithful actions of a transformation monoid that restrict to a given transitive and faithful action of its maximal subgroups at each idempotent of the minimal ideal.

The second part of the paper is motivated by the following quote by Steinberg in [25]:

"We hope that the theory of primitive permutation groups can be used to understand primitive transitive transformation monoids in the case the maximal subgroups of $\mathrm{I}(M)$ are non-trivial".

Theorems 38 and 39 respond to that hope and characterise the primitive transitive transformation monoids in terms of the primitive permutation representation of $G_{e}$ for all idempotent $e$ in the minimal ideal. These are improvements on the machinery used to study transitive transformation monoids. Among other applications, we prove that no non-trivial induced group action can be primitive, and if $G_{e}$ is a primitive permutation group there exists a unique action of $M$ that restricts to this action. This confirms the idea that the primitive transitive transformation monoids can be understood in terms of primitive groups in much the same way that irreducible representations of monoids can be understood in terms of irreducible representations of groups [10]. We also prove that for every transitive transformation monoid $M$ with maximal subgroup $G_{e}$ nilpotent, for an idempotent $e$ in the minimal ideal of $M$, then the monoid $M$ has a primitive action if and only if $M$ is a cyclic group of prime order. A last consequence on our study is that there is no primitive action of $M$ on a set $\Omega$ if $|\Omega e|=2$, for $e$ an idempotent in the minimal ideal $\mathrm{I}(M)$.

The paper aims at being largely self-contained, but it is assumed that the reader has some familiarity with basic semigroup theory results. The first section of the paper introduces basic concepts and classical results of transformation monoids. A basic study of the most important properties of the principal right ideal $e M$, for $e$ idempotent in $\mathrm{I}(M)$, is also presented there. Section 2 is devoted to the description of transitive transformation monoids. It contains our first main contribution; Theorems 17 and 20. This section also contains some applications of these results to specific transformation monoids. In section 3 we study primitive actions.

It contains our second main contribution; Theorems 38 and 39. Finally, we present a useful method to construct examples of primitive and transitive transformation monoids.

We believe that a good understanding of interesting examples is important in any algebraic theory. Thus, we have made a strong emphasis on this point throughout all sections.

\section{Preliminaries}

Unless otherwise stated, all semigroups and monoids here are finite. 


\subsection{Basic concepts}

An element $e$ of a semigroup $S$ is said to be idempotent if $e^{2}=e$. The set of idempotents of $S$ shall be denoted $\mathrm{E}(S)$. Note that $e S e$ is a monoid with identity $e$. The group of units of $e S e$ is denoted $G_{e}$ and is called the maximal subgroup of $S$ at $e$. If $s \in S$, then $s^{\omega}$ denotes the unique idempotent that is a positive power of $s$. Such power exists because of finiteness ([23. Appendix A]). A subset $I \subseteq S$ is said to be an ideal of $S$ if $S I S \subseteq I$. A semigroup $S$ is called simple if it has no proper ideals. A semigroup $S$ has a unique minimal ideal I $(S)$, which is a simple semigroup. For a semigroup $S$, we can always extend its structure to a monoid by adding an extra unit element. We denote it by $S^{1}=S \cup\{1\}$ where 1 is a new unit element if $S$ has no unit. If $S$ was already a monoid, then $S^{1}=S$. For an element $s$ in $S$, the set $S^{1} s S^{1}$ is the smallest ideal containing $s$. Moreover, for all $s \in \mathrm{I}(S)$, it holds $S^{1} s S^{1}=\mathrm{I}(S)$. If $s \in \mathrm{I}(S)$, then $s^{\omega} \in \mathrm{I}(S)$ and, thus, I $(S)$ contains idempotents.

From now on, we will only consider monoids.

A mapping $f: M \longrightarrow N$ between monoids $M$ and $N$ is a monoid homomorphism if

$$
\begin{aligned}
& (m n) f=(m) f(n) f, \quad \text { for all } m, n \in M \\
& \left(1_{M}\right) f=1_{N}, \quad \text { where } 1_{M} \text { and } 1_{N} \text { are the identities on } M \text { and } N, \text { respectively. }
\end{aligned}
$$

For a non-empty set $\Omega$, the monoid of all maps on $\Omega$ acting on the right is called the full transformation monoid of $\Omega$ and it is denoted by $\mathrm{T}_{\Omega}$. Furthermore, the group of units of $\mathrm{T}_{\Omega}$ is called the symmetric group of $\Omega$ and it is denoted by $\mathrm{S}_{\Omega}$. For a natural number $n$, the set $\{1, \cdots, n\}$ is denoted by $\mathrm{n}$. If the set $\Omega$ contains $n$ elements and the denotation of such elements is not relevant, we shall use the notation $T_{n}$ and $S_{n}$, respectively. A transformation $f$ in $\mathrm{T}_{\mathrm{n}}$ shall be denoted by an $n$-tuple $\left[a_{1}, \cdots, a_{n}\right]$ with $a_{i}=(i) f$ for all $i \in \mathrm{n}$. Hence, in $\mathrm{T}_{3}$, for example, the tuple $[1,2,2]$ represents the transformation $f$ defined by the equations (1) $f=1$, and $(2) f=(3) f=2$. For an element $f \in \mathrm{T}_{\mathrm{n}}$, the rank of $f$ is $|[\mathrm{n}] f|$.

An action of a monoid $M$ on a non-empty set $\Omega$ is a monoid homomorphism $\varphi$ from $M$ to $\mathrm{T}_{\Omega}$. In this case, we say that $\Omega$ is an $M$-set. To simplify the notation, if the action $\varphi$ is fixed, for $m \in M$ and $\alpha \in \Omega$ we will write $\alpha m$ instead of $(\alpha)(m) \varphi$. The $M$-set obtained by considering the action of $M$ on itself by right multiplication is called the regular $M$-set.

For an element $\alpha \in \Omega$, we define the stabiliser of $\alpha$ to be the $\operatorname{submonoid} \operatorname{Stab}_{M}(\alpha)=$ $\{m \in M \mid \alpha m=\alpha\}$. The action is faithful if the corresponding monoid homomorphism is injective. In this case $M$ can be seen as a submonoid of $\mathrm{T}_{\Omega}$, and we refer to the pair $(\Omega, M)$ as a transformation monoid.

A morphism $f: \Omega \longrightarrow \Gamma$ of $M$-sets is a map that preserves the action, that is $(\alpha m) f=$ $(\alpha) f m$, for all $\alpha \in \Omega$ and $m \in M$. If $f$ is a bijection, we say that the actions are equivalent. A non-empty subset $\Lambda \subseteq \Omega$ is $M$-invariant if $\Lambda M \subseteq \Lambda$. The subset $\Lambda$ inherits the action defined on $\Omega$. With this action, the inclusion map is an injective morphism of $M$-sets.

We say that a binary equivalence relation $\equiv$ defined on an $M$-set $\Omega$ is a congruence, if for all $\alpha, \beta \in \Omega$ and $m \in M, \alpha \equiv \beta$ implies $\alpha m \equiv \beta m$. If $\equiv$ is a congruence on $\Omega$, the quotient $\Omega / \equiv$ is an $M$-set for the action $[\alpha] m=[\alpha m]$, for all $\alpha \in \Omega$ and $m \in M$, and the quotient map from $\Omega$ to $\Omega / \equiv$ is a surjective morphism of $M$-sets.

As usual, the kernel of a morphism $f: \Omega \longrightarrow \Gamma$ of $M$-sets, defined as

$$
\operatorname{ker} f=\{(\alpha, \beta) \in \Omega \times \Omega \mid(\alpha) f=(\beta) f\}
$$

is a congruence on $\Omega$ and the image set 


$$
\operatorname{im} f=\{\beta \in \Gamma \mid \text { there exists some } \alpha \in \Omega \text { with }(\alpha) f=\beta\}
$$

is an $M$-invariant subset of $\Gamma$. Moreover, the actions of $M$ on $\Omega / \operatorname{ker} f$ and $\operatorname{im} f$ are equivalent.

An $M$-invariant subset of the form $\alpha M$, for $\alpha \in \Omega$, is called cyclic. A cyclic subset of $M$ is called minimal if it does not contain any proper cyclic subset. Cyclic sets allow us to define an equivalence relation on $\Omega$. We say that two elements $\alpha, \beta \in \Omega$ are related if $\alpha M=\beta M$. The classes of this equivalence relation are called orbits. The action of $M$ is called transitive if it has a single orbit. Equivalently, the action is transitive if, for every $\alpha, \beta \in \Omega$, there exists $m, n \in M$ with $\alpha m=\beta$ and $\beta n=\alpha$.

For example, the inclusion map define actions of $T_{n}$ and $S_{n}$ on $n$. These actions, which are called natural, are faithful and transitive.

\subsection{Preliminary Results}

One of the main goals in this paper is to characterise transitive monoid actions. This problem is completely solved in the group case. If a group $G$ has a transitive action on a set $\Omega$, then the action of $G$ on $\Omega$ is equivalent to the action of $G$ by right multiplication on the right coset space of a subgroup $H$, defined as the stabiliser of an element. The kernel of this action is called the core of $H$ in $G$, denoted $\operatorname{Core}_{G}(H)$, and is the largest normal subgroup of $G$ contained in $H$. We say that $H$ is core-free in $G$ if $\operatorname{Core}_{G}(H)=1$. Consequently, the study of transitive permutation groups $G$ is equivalent to the study of core-free subgroups of $G$.

As expected, the monoid case is not so transparent but we can still prove some interesting structural results. The following propositions have been included for the sake of completeness. See [11, Chapter 10] for a general overview on the subject. Generalizations to representations by partial mappings can be found in [21].

Proposition 1. The action by right multiplication of a monoid $M$ on $m M$, with $m \in M$, is transitive if and only if $m M$ is minimal. Moreover, $\mathrm{I}(M)$ is the set of all $m \in M$ with $m M$ minimal.

Proof. If $n M \subseteq m M$ and the action is transitive, it necessarily holds that $n M=m M$. On the converse, if $p, q \in m M$ then $p M, q M \subseteq m M$. Minimality of $m M$ yields $p M=q M=m M$.

Let $m \in \mathrm{I}(M)$ and $x \in m M$. Then $x M \subseteq m M$. Since $x \in \mathrm{I}(M)$, it follows that $M x M=M m M$. Then $m=u x v$ for some $u, v \in M$. This means that $m M$ is contained in $u x M$. Since $|u x M| \leq|x M|$, we have that $x M=m M$ and $m M$ is minimal. Conversely, suppose that $m M$ is minimal for some $m \in M$. Let $n \in \mathrm{I}(M)$, then $m n \in \mathrm{I}(M)$. Since $m n M$ is contained in $m M$, minimalily of $m M$ yields $m n M=m M$. Therefore $m \in \mathrm{I}(M)$.

Since $\mathrm{I}(M)$ contains idempotents, we will use idempotents instead of arbitrary elements from $\mathrm{I}(M)$.

Proposition 2. Let $M$ be a monoid and $e \in \mathrm{E}(\mathrm{I}(M))$. If $M$ has a transitive and faithful action on a non-empty finite set $\Omega$, then the action of $M$ on eM by right multiplication is transitive and faithful.

Proof. Let $e$ be an idempotent in I $(M)$. By Proposition 1, $M$ acts transitively on $e M$ by right multiplication. Let $\alpha$ be an element in $\Omega e$ and consider the morphism of $M$-sets : $e M \longrightarrow \Omega$, given by $m \longmapsto \alpha m$. Assume that $m, n \in M$ satisfy that epm $=e p n$ for all $p \in M$. It follows that $\alpha(p m)=\alpha(p n)$ for all $p \in M$. On the other hand, let $\beta$ be an element in $\Omega$, as the 
action on $\Omega$ is transitive there exists an element $q \in M$ with $\beta=\alpha q$. It follows that for all $\beta \in \Omega, \beta m=\alpha(q m)=\alpha(q n)=\beta n$. Finally, $m=n$ as the action on $\Omega$ is faithful.

Since the action on $\Omega$ is transitive, it follows that the map constructed above is surjective. As a consequence, we have:

Corollary 3. Any transitive and faithful action of a monoid $M$ on a finite non-empty set $\Omega$ is equivalent to the action of $M$ on some quotient $e M / \equiv$ by right multiplication, where $e$ is an idempotent on $\mathrm{I}(M)$ and $\equiv$ is a right congruence on the $M$-set eM.

Note that the action of $M$ on $e M$ is the right Schützenberger representation, since $e M$ coincides with the $\mathcal{R}$-class of $e$ [21]. Corollary 3 highlights the need to develop a structural study of the principal right ideals $e M$, for $e$ idempotent in $\mathrm{I}(M)$. The next two results provide useful information in this direction. The first one describes the maximal subgroup of $M$ at these idempotents and it states that, up to equivalence, there is a unique action of $M$ by right multiplication on such minimal cyclic sets.

Proposition 4 ([25, Proposition 3.3]). Let $M$ be a monoid and e, $f \in \mathrm{E}(\mathrm{I}(M))$. Then:

i) $G_{e}=e M e$.

ii) $e M$ and $f M$ are equivalents as $M$-sets.

iii) If $f$ is an idempotent in $\mathrm{I}(M)$, then $G_{e}$ and $G_{f}$ are isomorphic.

Note that $e M$ is contained in $\mathrm{I}(M)$ for all $e \in \mathrm{E}(\mathrm{I}(M))$. However, the equality does not hold in general as the following example shows.

Example 5. Let $M$ be the monoid consisting on two left zeroes $e$ and $f$ and an identity element. Hence, the ideal $\mathrm{I}(M)$ is given by the set $\{e, f\}$, which also coincides with the idempotent elements in $\mathrm{E}(\mathrm{I}(M))$. The corresponding cyclic subset of $e$ is given by the principal right ideal $e M=\{e\}$, which does not contain $f$.

According to [25, Proposition 3.3], I $(M)$ is a disjoint union of the maximal subgroups $G_{e}$, with $e \in \mathrm{E}(\mathrm{I}(M))$. The following theorem is crucial for analysing transitive transformation monoids. It shows that the principal right ideal $e M$, with $e \in \mathrm{E}(\mathrm{I}(M))$, are disjoint unions of isomorphic maximal subgroups as well. The next result easily follows from Rees's Theorem and Green's Lemma [6]. We include its proof for the sake of completeness.

Theorem 6. Let $e$ be an idempotent in $\mathrm{I}(M)$. Then:

i) The classes $\left\{G_{e} m \mid m \in M\right\}$ form a partition of $e M$;

ii) $G_{e} m$ has exactly $\left|G_{e}\right|$ elements;

iii) If $f$ is an idempotent in $e M$, then $f e=e$ and $e f=f$. Moreover, for all $g \in G_{e}, f g=g$;

iv) If $f$ is an idempotent in eM, it holds $G_{e} f=f M f=G_{f}$;

v) $G_{e} m$ has exactly one idempotent. 
Proof. Let $g, h \in G_{e}, m, n \in M$ and $f \in \mathrm{E}(\mathrm{I}(M))$. If $e n \in G_{e} m$, then $G_{e} n \subseteq G_{e} m$ and $e n=g m$ for some $g \in G_{e}$. Hence $g^{-1} n=g^{-1} g m=e m$. Thus, for all $h \in G_{e}$, we have that $h m=h e m=h g^{-1} n \in G_{e} n$. Therefore $G_{e} n=G_{e} m$ and item i) follows. For item ii), if $g m=h m$, we derive the equation geme $=$ heme in $G_{e}=e M e$. It follows $g=h$. Hence $G_{e} m$ has exactly $\left|G_{e}\right|$ elements. For item iii), suppose that $f \in e M$. Since $G_{e}=e M e$, we have that $f e \in \mathrm{E}\left(G_{e}\right)$. Thus, $f e=e$ and $e f=f$. For every element $g \in G_{e}$, we have $f g=f(e g)=e g=g$. For item iv), assume that $f \in e M$. Then $G_{e} f=f e M e f \subseteq f M f$. On the converse, $f M f=e f M e f \subseteq G_{e} f$. Finally, for item v), note that $e m \in G_{e} m$ and for every natural number $k>1$, $(e m)^{k}=\left[(e m)^{k-1} e\right] m \in G_{e} m$. It follows that $f=(e m)^{\omega} \in \mathrm{E}\left(G_{e} m\right)$. Then $G_{e} m=G_{e} f$. Assume there exists some $g \in G_{e}$ such that $g f$ idempotent. Then $g f g f e=g f e$. Since, by iii), $f e=e$, it follows that $g f g=g$. Hence $g f e=g f g g^{-1}=g g^{-1}=e$. Consequently $g f e=e$. By iii), $f e=e$. Hence $g=e$ and $g f=f$. Therefore $G_{e} m$ has exactly one idempotent.

The sets $G_{e} m$ are precisely the $\mathcal{H}$-classes of $e M[6]$. The last theorem can be summarised in the following equation

$$
e M=\biguplus_{f \in \mathrm{E}(e M)} G_{f}
$$

being all groups $G_{f}$, for $f \in \mathrm{E}(e M)$, isomorphic to $G_{e}$. Note that the action of $M$ by right multiplication on $e M$ transforms $\mathcal{H}$-classes into $\mathcal{H}$-classes. This action is transitive but it is not necessarily faithful as the next example shows.

Example 7. Consider any non-trivial group $G$ whose identity element shall be denoted by $e$. For a natural number $n$, we denote the minimum of $i, j \in \mathrm{n}$ by $\min (i, j)$. The set $M=G \times \mathrm{n}$ is a monoid with the operation

$$
(g, i)(h, j)=(g h, \min (i, j))
$$

with $g, h \in G$ and $i, j \in \mathrm{n}$, and unit element $(e, n)$. It is straightforward to see that $\mathrm{I}(M)=$ $\{(g, 1) \mid g \in G\} \cong G$. The element $(e, 1)$ is the unique idempotent in $\mathrm{I}(M)$. Moreover, the principal right ideal $(e, 1) M$ coincides with $\mathrm{I}(M)$. The action of $M$ on $\mathrm{I}(M)$ is transitive. However, for $n \geq 2$, the action is not faithful.

Two interesting particular cases arise. We can consider the case where the maximal subgroup $G_{e}$ of the minimal ideal $\mathrm{I}(M)$ is trivial and the case where $\mathrm{I}(M)$ is a group. In the first one, $e M$ is a union of idempotents and, in the second one, I $(M)$ has exactly one idempotent $e$ and $\mathrm{I}(M)=e M=G_{e}$.

Corollary 8. Let $(\Omega, M)$ be a transitive transformation monoid.

i) [25, Proposition 3.13] If $G_{e}$ is trivial for some e $\in \mathrm{E}(\mathrm{I}(M))$, then $\mathrm{I}(M)=e M, \Omega$ and eM are isomorphic $M$-sets, and $\mathrm{I}(M)$ is the set of constant maps on $\Omega$.

ii) If e $M$ is a group for some $e \in \mathrm{E}(\mathrm{I}(M))$, then $M$ is a group. In particular, every transitive transformation inverse monoid is a group.

Proof. For item i), assume that $G_{e}$ is trivial for some $e \in \mathrm{E}(\mathrm{I}(M))$. Then $e M e=\{e\}$ and $G_{f}$ is trivial for all $f \in \mathrm{E}(\mathrm{I}(M))$ by Proposition 4. By Theorem 6, $e M$ is a set of idempotents on which $M$ acts transtively and faithfully by right multiplication. Let $\alpha$ be an element in $\Omega e$. 
Then $\alpha e=\alpha$. If $\beta \in \Omega$, there exists $m \in M$ such that $\beta=\alpha m$ by transitivity. Therefore, $\beta e=\alpha m e=\alpha e m e=\alpha e=\alpha$. Hence $e$ is a constant map on $\Omega$, and the same is true for all $f \in \mathrm{E}(\mathrm{I}(M))$. In particular, $f=e f$ and so $\mathrm{I}(M)=e M$. Since the congruence in Corollary 3 is the identity, it follows that $\Omega$ and $e M$ are isomorphic $M$-sets. Finally, for item ii), suppose that $e M$ is a group. Then, the unique idempotent in $e M$ is exactly $e$. By Theorem 6 , $e M=G_{e}$. The monoid $M$ acts transitively and faithfully on $e M$ by Proposition 2. Since $e m e=e m=e m 1$ for all $m \in M$, it follows that $e=1$ and $e M=M=G_{e}=\mathrm{I}(M)$.

To this matter recall that, if $M$ is a transitive transformation inverse monoid, then $\mathrm{I}(M)$ is a group $([6],[23])$.

Example 9. Consider the full transformation monoid on $n$ elements $\mathrm{T}_{\mathrm{n}}$. For $j \in \mathrm{n}$, denote by $\varsigma_{j}$ the constant mapping to $j$, that is $(k) \varsigma_{j}=j$ for all $k \in \mathrm{n}$. The set $\zeta_{\mathrm{n}}=\left\{\varsigma_{j} \mid j \in \mathrm{n}\right\}$ is an ideal in $\mathrm{T}_{\mathrm{n}}$. In fact, for $f \in \mathrm{T}_{\mathrm{n}}$, we have:

$$
f \varsigma_{j}=\varsigma_{j}, \quad \varsigma_{j} f=\varsigma_{(j) f} .
$$

Clearly $\mathrm{I}\left(\mathrm{T}_{\mathrm{n}}\right)=\zeta_{\mathrm{n}}$ and $G_{e}=\{e\}$ for all $e \in \mathrm{E}\left(\mathrm{I}\left(\mathrm{T}_{\mathrm{n}}\right)\right)$.

On the other hand, it is clear that the identity morphism defines an action of $T_{n}$ on $n$ which is transitive and faithful. This action is called natural. By Corollary $8, \mathrm{I}\left(\mathrm{T}_{\mathrm{n}}\right)=\varsigma_{j} \mathrm{~T}_{\mathrm{n}}$ for all $\varsigma_{j} \in \mathrm{I}\left(\mathrm{T}_{\mathrm{n}}\right)$, and the action of $\mathrm{T}_{\mathrm{n}}$ on $\mathrm{I}\left(\mathrm{T}_{\mathrm{n}}\right)$ by right multiplication is equivalent to the natural action. It shows an striking difference with $\mathrm{S}_{\mathrm{n}}$ : any transitive and faithful action of $\mathrm{T}_{\mathrm{n}}$ on a non-empty finite set is equivalent to the natural action (see [11, Theorem 10.3.1]).

Example 10. Let $\mathrm{T}_{3}$ be the full transformation monoid on 3 elements. Let $N$ be the subset of $\mathrm{T}_{3}$ containing all transformations whose image belongs to the set $2 . N$ is a subsemigroup of $\mathrm{T}_{3}$. Consider the monoid $M=N^{1}$. Clearly the action of $N$ on 3 is not transitive. Then $\mathrm{I}(M)=\left\{\varsigma_{1}, \varsigma_{2}\right\}=\varsigma_{1} M$, and so $G_{e}$ is trivial for any idempotent $e \in \mathrm{I}(M)$. However, the action of $M$ on $\mathrm{I}(M)$ is transitive but it is not faithful, as the mappings $f$ and $g$ given by

$$
f=[1,2,1], \quad g=[1,2,2]
$$

satisfy that $f \neq g$, but they have the same action on the elements of $\mathrm{I}(M)$.

\subsection{A wreath product construction}

For a monoid $M$ having a transitive and faithful action on a set $e M$ we can better describe the structure of $M$ through an embedding into a wreath product which heavily depends on the partition of the right ideals $e M$ studied before. Basic concepts about wreath products are introduced in order to keep the paper self-contained. The work presented in this subsection can be found in [2, 23]. The reader may consult [9, 15] for further results in group theory.

Let $G$ be a group and let $D$ be a monoid acting on a finite set of $n$ elements. We can assume this set is $\mathrm{n}=\{1, \cdots, n\}$. Consider the group $G^{\mathrm{n}}$ of all functions from $\mathrm{n}$ to $G$ on which the monoid $D$ has an action given by $(j)(\psi m)=(j m) \psi$, with $m \in D, j \in \mathrm{n}$ and $\psi \in G^{\mathrm{n}}$. We define a binary operation on the set $G^{\mathrm{n}} \times D=\{(\psi, m) \mid \psi: \mathrm{n} \longrightarrow G, m \in D\}$ as follows

$$
(\psi, m)\left(\psi^{\prime}, m^{\prime}\right)=\left(\varphi, m m^{\prime}\right), \quad \text { where }(j) \varphi=(j) \psi(j m) \psi^{\prime} .
$$

Then it is straightforward to verify that, with respect to this binary operation, the set $G^{\mathrm{n}} \times D$ is a monoid in which $\left(\psi_{e}, 1\right)$ is the identity element, where $e$ is the identity of $G, 1$ is 
the identity of $D$ and $(j) \psi_{e}=e$ for all $j \in \mathrm{n}$. In general, we shall use $\psi_{g}$ to denote the map $(j) \psi_{g}=g$, for all $j \in \mathrm{n}$. We call $G^{\mathrm{n}} \times D$ the wreath product of $G$ with $D$ with respect to the aforementioned action and we denote this monoid by $G 2_{\mathrm{n}} D$. It is also straightforward to see that the projection $(\psi, m) \longmapsto m$ is a surjective monoid homomorphism from $G \ln D$ to $D$.

Let $M$ be a monoid and let $e$ be an idempotent in $\mathrm{I}(M)$. Assume that the action of $M$ on $e M$ by right multiplication is faithful and transitive. By Theorem $6,\left\{G_{e} m \mid m \in M\right\}$ is a partition of $e M$. Let $\mathrm{E}(e M)=\left\{e, e_{2}, \cdots, e_{n}\right\}$ be the set of all idempotents of $e M$. Then $M$ acts on $\mathrm{E}(e M)$ in the following way: for $m \in M$ and $e_{j}$ in $\mathrm{E}(e M), e_{j} m$ is defined to be the unique idempotent in $G_{e}\left(e_{j} m\right)$. This action is transitive, but it is not faithful in general (see Example 7). Let $\bar{M}$ be the quotient monoid of $M$ over the kernel of the morphism $M \longrightarrow \mathrm{T}_{\mathrm{n}}$ corresponding to the above action. This monoid is called Right Letter Mapping of $M$ in [2, 23, 25. Clearly $\bar{M}$ has a transitive and faithful action on $\mathrm{n}$. Moreover, by Theorem 6 , $\overline{e_{j}}$ acts as the constant function $\varsigma_{j}$ on $\mathrm{n}$ for all idempotent $e_{j}$ in $e M$, and every maximal subgroup $G_{\overline{e_{j}}}$ is trivial.

The following proposition is a particular case of [25, Corollary 3.18]. We have decided to include a proof since it turns out to be crucial in our study of transitive transformation monoids.

Proposition 11. The monoid $M$ can be embedded in $G_{e} \mathrm{ln}_{\mathrm{n}} \bar{M}$.

Proof. We use the notation introduced above. Let $m \in M$ and $e_{i} \in \mathrm{E}(e M)$. Then, by Theorem 6, there exists a unique element $g \in G_{e}$ such that $e_{i} m=g e_{i^{\prime}}$ for some idempotent $e_{i^{\prime}}$ in $e M$. We denote the element $g$ by $(i) \rho_{m}$ since only depends on $e_{i}$ and $m$. To simplify notation we will denote the idempotent $e_{i}$ by the symbol $i$. Hence we have a map $\rho_{m}: \mathrm{n} \longrightarrow G_{e}$. We denote the image of $m$ in $\bar{M}$ by $\bar{m}$. We define a map $\phi: M \longrightarrow G_{e} \imath_{n} \bar{M}$ by $(m) \phi=\left(\rho_{m}, \bar{m}\right)$.

Let $m^{\prime} \in M$. We have $e_{j} m m^{\prime}=g_{1} e_{j^{\prime}}$ and $e_{j} m=g_{2} e_{j^{\prime \prime}}$ where $g_{1}, g_{2} \in G_{e}$ and $e_{j^{\prime}}, e_{j^{\prime \prime}} \in$ $\mathrm{E}(e M)$. Then $(j) \rho_{m m^{\prime}}=g_{1}$ and $(j) \rho_{m}=g_{2}$. It follows $g_{1} e_{j^{\prime}}=e_{j} m m^{\prime}=g_{2} e_{j^{\prime \prime}} m^{\prime}$ and so $e_{j^{\prime \prime}} m^{\prime}=g_{2}^{-1} g_{1} e_{j^{\prime}}$. Therefore $\left(j^{\prime \prime}\right) \rho_{m^{\prime}}=(j m) \rho_{m^{\prime}}=g_{2}^{-1} g_{1}$. Hence $(j) \rho_{m m^{\prime}}=(j) \rho_{m}(j m) \rho_{m^{\prime}}$. It proves that $\phi$ is a monoid homomorphism.

Suppose $\left(\rho_{m}, \bar{m}\right)=\left(\rho_{m^{\prime}}, \bar{m}^{\prime}\right)$. Let $p$ be an arbitrary element in $e M$, then $p=g e_{j}$ for some $g \in G_{e}$ and $e_{j} \in \mathrm{E}(e M)$. As $\bar{m}=\bar{m}^{\prime}$, the cosets $G_{e}\left(e_{j} m\right)$ and $G_{e}\left(e_{j} m^{\prime}\right)$ are equal. Therefore, there exist an idempotent $e_{j^{\prime}} \in \mathrm{E}(e M)$ and elements $g_{1}, g_{2} \in G_{e}$ with $g\left(e_{j} m\right)=g_{1} e_{j^{\prime}}$ and $g\left(e_{j} m^{\prime}\right)=g_{2} e_{j^{\prime}}$. Thus, $(j) \rho_{m}=g^{-1} g_{1}$ and $(j) \rho_{m^{\prime}}=g^{-1} g_{2}$. Since $(j) \rho_{m}=(j) \rho_{m^{\prime}}$, it follows that $g_{1}=g_{2}$. Hence $p m=p m^{\prime}$ for all $p \in e M$. Since the action is faithful, we have $m=m^{\prime}$. Consequently, $\phi$ is a monomorphism.

Note that $M$ can also be embedded in $G_{f} \imath_{\mathrm{n}} \bar{M}$ for any other idempotent $f \in \mathrm{E}(\mathrm{I}(M))$ and it would immediately follow from Proposition 4 that this two embeddings would be isomorphic. With the embedding considered in Proposition 11 we can better describe elements of $e M$ as elements in the wreath product. Let $p \in e M$. Then there exist elements $g \in G_{e}$ and $e_{j} \in \mathrm{E}(e M)$ such that $p=g e_{j}$. Moreover, $e_{j^{\prime}} p=\left(e_{j^{\prime}} g\right) e_{j}=g e_{j}$ for every $e_{j^{\prime}} \in \mathrm{E}(e M)$ by Theorem 6 iii). It follows that $\bar{p}$ corresponds to $\varsigma_{j}$, the constant mapping to $j$ in $\mathrm{T}_{\mathrm{n}}$, and $\rho_{p}=\psi_{g}$. Therefore $[e M] \phi=\left\{\left(\psi_{g}, \varsigma_{j}\right) \in G_{e} \imath_{\mathrm{n}} \bar{M} \mid g \in G_{e}, j \in \mathrm{n}\right\}$. Now, if $\left(\psi_{g}, \varsigma_{j}\right)$ is an element in $[e M] \phi$ and $\left(\rho_{m}, \bar{m}\right)$ is an element in $[M] \phi$, the multiplication is given by

$$
\left(\psi_{g}, \varsigma_{j}\right)\left(\rho_{m}, \bar{m}\right)=\left(\psi_{g\left((j) \rho_{m}\right)}, \varsigma_{j}\right) .
$$

Let $n$ be a natural number and let $N$ be a submonoid of $\mathrm{T}_{\mathrm{n}}$ containing all constant mappings $\left\{\varsigma_{j} \mid j \in \mathrm{n}\right\}$. Then $\mathrm{I}(N)=\mathrm{I}\left(\mathrm{T}_{\mathrm{n}}\right)=\left\{\varsigma_{j} \mid j \in \mathrm{n}\right\}$. Furthermore, let $G$ be an arbitrary 
group whose identity element shall be denoted by $e$. We define the following subset of the monoid $G \ln N$

$$
L=\left\{\left(\psi_{g}, \varsigma_{j}\right) \mid g \in G, j \in \mathrm{n}\right\} .
$$

A simple calculation shows that the equation $\left(\psi_{g}, \varsigma_{j}\right)\left(\psi_{h}, \varsigma_{j^{\prime}}\right)=\left(\psi_{g h}, \varsigma_{j^{\prime}}\right)$ holds for all pairs $g, h \in G$ and $j, j^{\prime} \in \mathrm{n}$. Thus, $L$ is a subsemigroup of $G l_{\mathrm{n}} N$. It is straightforward to see that $L$ is contained in the minimal ideal $\mathrm{I}\left(G 2_{n} N\right)$. The set of all the idempotent elements in $L$ is given by $\mathrm{E}(L)=\left\{\left(\psi_{e}, \varsigma_{j}\right) \mid j \in \mathrm{n}\right\}$ which is bijective to $\mathrm{n}$. Moreover, fixing some element $j$ in n, the idempotent $\widehat{e}_{j}=\left(\psi_{e}, \varsigma_{j}\right)$ in $L$ satisfies that $G_{\widehat{e}_{j}}=\left\{\left(\psi_{g}, \varsigma_{j}\right) \mid g \in G\right\}$ which is isomorphic to $G$. It also holds that $\widehat{e}_{j}\left(G l_{\mathrm{n}} N\right)=L$.

Combining some of the above results, we have:

Theorem 12. A monoid $M$ is a transitive transformation monoid if and only if there exist a group $G$, a natural number $n$ and a submonoid $N$ of $\mathrm{T}_{\mathrm{n}}$ containing all constant mappings $\mathrm{I}\left(\mathrm{T}_{\mathrm{n}}\right)$, such that $M$ is isomorphic to a submonoid of the wreath product $G 2_{\mathrm{n}} N$ containing the subsemigroup $L=\left\{\left(\psi_{g}, \varsigma_{j}\right) \mid g \in G, j \in \mathrm{n}\right\}$.

Proof. Bearing in mind Proposition 11 and the subsequent discussions, it is clear that only the sufficency of the condition is in doubt. Assume that $M$ is a submonoid of $G \mathrm{n}_{\mathrm{n}} N$ containing $L$. If $j$ is an element in $\mathrm{n}$, the idempotent $\widehat{e}_{j}=\left(\psi_{e}, \varsigma_{j}\right)$ of $L$ satisfies $\widehat{e}_{j} M=L$. Consider the action of $M$ given by right multiplication on the subsemigroup $L$. As $\widehat{e}_{j}$ is an idempotent in the minimal ideal, the action is transitive by Proposition 1. Let $(\xi, p),\left(\xi^{\prime}, p^{\prime}\right)$ be two elements in $M$ such that

$$
\left(\varphi, \varsigma_{j p}\right)=\left(\psi_{g}, \varsigma_{j}\right)(\xi, p)=\left(\psi_{g}, \varsigma_{j}\right)\left(\xi^{\prime}, p^{\prime}\right)=\left(\varphi^{\prime}, \varsigma_{j p^{\prime}}\right) \text {, for all } g \in G \text { and } j \in \mathrm{n} .
$$

Then $(k) \varphi=(k) \psi_{g}\left(k \varsigma_{j}\right) \xi=g(j) \xi$ and $(k) \varphi^{\prime}=(k) \psi_{g}\left(k \varsigma_{j}\right) \xi^{\prime}=g(j) \xi^{\prime}$, for all $k \in \mathrm{n}$. Hence, $\xi=$ $\xi^{\prime}$ and $p=p^{\prime}$. Thus, the action is faithful and $M$ is a transitive transformation monoid.

\section{Transitive and faithful monoid actions}

The main goal in this section is to describe all transitive and faithful actions of a monoid. The following simple result provides the key to the study of transitive and faithful actions.

Lemma 13 ([25, Corollary 2.11]). Let $M$ be a monoid and $e \in \mathrm{E}(M)$. Every transitive and faithful action of $M$ on a set $\Omega$ restricts to a transitive and faithful action of the monoid eMe on $\Omega e$.

An important fact about irreducible representations of a semigroup is that they can be parameterised in terms of the irreducible representations of its maximal subgroups. This is a consequence of the results of Clifford, Munn and Ponizovsky (see [6, Chapter 5]).

Our main objective in this section is to parameterise the transitive and faithful actions of a monoid $M$ in terms of transitive and faithful actions of the maximal subgroups at each idempotent in $\mathrm{I}(M)$.

We begin with a preparatory result that is a direct consequence of Theorem 6. The first part of this theorem can be found on [11, Theorem 10.4.1]. We have include it for the sake of completeness. 
Proposition 14. Let $M$ be a monoid and let e be an idempotent in $\mathrm{I}(M)$. If $H$ is a subgroup of $G_{e},\left\{x_{1}, \cdots, x_{r}\right\}$ is a transversal of $H$ in $G_{e}$ and $\left\{e, e_{2} \cdots, e_{n}\right\}$ is the set of idempotents of eM, then

1. The set $\mathbb{H}=\left\{H x_{i} e_{j} \mid 1 \leq i \leq r, 1 \leq j \leq n\right\}$ forms a partition of eM. The monoid $M$ acts on $\mathbb{H}$ by right multiplication and this action is transitive.

2. Every transitive action of $M$ is equivalent to a transitive action of $M$ on a quotient of an $M$-set $\mathbb{H}$.

3. Every transitive and faithful action of $M$ is equivalent to a transitive action of $M$ on a quotient of an $M$-set $\mathbb{H}$ with $\operatorname{Core}_{G_{e}}(H)=1$.

Proof. If $\left\{x_{1}, \cdots, x_{r}\right\}$ is a transversal of $H$ in $G_{e}$, then $\left\{H x_{i} \mid 1 \leq i \leq r\right\}$ is a partition of $G_{e}$. By Theorem 6 , if $\left\{e, e_{2} \cdots, e_{n}\right\}$ is the set of idempotents of $e M$, then $\left\{G_{e} e_{j} \mid 1 \leq j \leq n\right\}$ is a partition of $e M$, and every $G_{e} e_{j}$ is bijective with $G_{e}$. Consequently $\mathbb{H}=\left\{H x_{i} e_{j} \mid 1 \leq\right.$ $i \leq r, 1 \leq j \leq n\}$ is a partition of $e M$ of $r n$ elements. It is clear that $M$ acts on $\mathbb{H}$ by right multiplication. This action is transitive because the transitivity of the action of $M$ on $e M$.

Assume that $M$ has a transitive action on $\Omega$. Consider an element $\alpha \in \Omega$ and the mapping from $e M$ to $\Omega$ that sends $e m \in e M$ to $\alpha e m$. This mapping is a surjective morphism of $M$-sets. Moreover, for the subgroup $H=\{h \in e M e \mid \alpha h=\alpha\}$ of $G_{e}$, we have that the associated partition $\mathbb{H}$ lies in the kernel of this morphism. This proves Statement 2.

Assume now that the action described in Statement 2 is faithful. Then the restriction of the action of $e M e$ on $\Omega e$ is faithful by Lemma 13 . Consequently, the stabilizer of $\alpha e$ is a core-free subgroup of $G_{e}$.

According to Corollary 3 and following the results presented in Proposition 14, the description of the right-congruences on the $M$-set $\mathbb{H}$ is of interest.

Definition 15. Bearing in mind the notation introduced in Proposition 14 and the partition described there, we define an equivalence relation $\simeq^{i}$, for each $i \in\{1, \cdots r\}$, in the set $\mathrm{n}=$ $\{1, \cdots n\}$ as follows:

$$
j \simeq^{i} j^{\prime} \Leftrightarrow H x_{i}(j) \rho_{m}=H x_{i}\left(j^{\prime}\right) \rho_{m} \text { for all } m \in M,
$$

where $\rho_{m}$ is the map introduced in the proof of Proposition 11. Recall that $(j) \rho_{m}=e_{j} m e$.

Note that this family of equivalence relations does not depend on the choice of the transversal. Moreover, another choice of a minimal idempotent $f$ in $\mathrm{I}(M)$ would lead to the same definition up to an ordering in the set of indices. That is, the family of equivalence relations only depends on the given subgroup.

Assume that a monoid $M$ has a transitive and faithful action on a set $\Omega$. Let $e \in \mathrm{E}(\mathrm{I}(M))$. By Proposition 2, $M$ has a transitive and faithful action on $e M$ by right multiplication. If we consider a submonoid of a wreath product as in Theorem 12 , the last family of equivalence relations can be further described as follows.

Proposition 16. Let $G$ be a group with identity e, let $n$ be a natural number, and let $N$ be a submonoid in $\mathrm{T}_{\mathrm{n}}$ containing all constant functions $\mathrm{I}\left(\mathrm{T}_{\mathrm{n}}\right)$. Let $M$ be a submonoid of the wreath product monoid $G \ln _{\mathrm{n}} N$ containing the subsemigroup $L=\left\{\left(\psi_{g}, \varsigma_{j}\right) \mid g \in G, j \in \mathrm{n}\right\}$. Let $H$ be a subgroup of $G$ and fix some transversal $\left\{x_{1}, \cdots, x_{r}\right\}$ of $H$ in $G$, then

$$
j \simeq^{i} j^{\prime} \Leftrightarrow \text { for all }(\psi, p) \in M, H x_{i}(j) \psi=H x_{i}\left(j^{\prime}\right) \psi .
$$


Proof. From Proposition 11, we have the following equations $\left(\psi_{e}, \varsigma_{j}\right)(\psi, p)=\left(\psi_{(j) \psi}, \varsigma_{j p}\right)=$ $\left(\psi_{(j) \psi}, \varsigma_{1}\right)\left(\psi_{e}, \varsigma_{j p}\right)$.

Theorem 17. Let $M$ be a monoid having a transitive action on a set $\Omega$ such that the restricted action of $G_{e}$ on $\Omega e$ is equivalent to the action of $G_{e}$ on the right cosets of a subgroup $H$ of $G_{e}$. Let $T=\left\{x_{1}, \cdots, x_{r}\right\}$ be a right transversal of $H$ in $G_{e}$ and let $\left\{e, e_{2} \cdots, e_{n}\right\}$ be the set of idempotents of eM. The right congruence $\equiv$ that defines the $M$-set $\Omega$, is a right congruence on the set $\mathbb{H}=\left\{H x_{i} e_{j} \mid 1 \leq i \leq r, 1 \leq j \leq n\right\}$ satisfying the following statements:

i) If $H x_{i} e_{j} \equiv H x_{i^{\prime}} e_{j^{\prime}}$, i.e, $H x_{i} e_{j}$ and $H x_{i^{\prime}} e_{j^{\prime}}$ are both contained in the same $\equiv$-class, then $i=i^{\prime}$ and $j \simeq^{i} j^{\prime}$;

ii) If $H x_{i} e_{j} \equiv H x_{i} e_{j^{\prime}}$ and $m \in M$, then $H x_{k}\left(e_{j \bar{m}}\right) \equiv H x_{k}\left(e_{j^{\prime} \bar{m}}\right)$, with $x_{i}(j) \rho_{m} \in H x_{k}$.

Every equivalence relation on the set $\mathbb{H}$ satisfying Statements i) and ii) is a congruence on the $M$-set $\mathbb{H}$ whose action is transitive and whose restriction to $G_{e}$ is equivalent to the action of $G_{e}$ on the partition $\left\{H x_{i} \mid 1 \leq i \leq r\right\}$.

Proof. Since the action restricted to $G_{e}$ on $\Omega_{e}$ is equivalent to the action of $G_{e}$ on the right cosets of $H$, there exists $\alpha \in \Omega e$ such that $H=\left\{h \in G_{e} \mid \alpha h=\alpha\right\}$. By the proof of the Statement 2 of Proposition 14, the right-congruence $\equiv$ defining the $M$-set $\Omega$ is a rightcongruence on the set $\mathbb{H}$.

Since the restricted action of $G_{e}$ on $\Omega e$ is equivalent to the action of $G_{e}$ on the right cosets of $H \leq G_{e}$, we have that $H x_{i}=H x_{i} e \equiv H x_{i^{\prime}} e=H x_{i^{\prime}}$ if and only if $i=i^{\prime}$.

Assume $H x_{i} e_{j} \equiv H x_{i^{\prime}} e_{j^{\prime}}$. Then $H x_{i} e_{j} e \equiv H x_{i^{\prime}} e_{j^{\prime}} e$ that is $H x_{i} \equiv H x_{i^{\prime}}$ and $i=i^{\prime}$. Moreover, let $m \in M, H x_{i} e_{j} m=H x_{i}(j) \rho_{m}\left(e_{j \bar{m}}\right)$ and $H x_{i} e_{j^{\prime}} m=H x_{i}\left(j^{\prime}\right) \rho_{m}\left(e_{j^{\prime} m}\right)$ are contained in the same $\equiv$-class and arguing as before, we have that $H x_{i}(j) \rho_{m}=H x_{i}\left(j^{\prime}\right) \rho_{m}$, that is, $j \simeq^{i} j^{\prime}$. This proves Property i).

Let $k \in\{1, \cdots, r\}$ be such that $x_{i}(j) \rho_{m} \in H x_{k}$. Then $H x_{k}\left(e_{j \bar{m}}\right) \equiv H x_{k}\left(e_{j^{\prime} \bar{m}}\right)$ and Property ii) holds.

Conversely, an equivalence relation satisfying Statements i) and ii) is a right congruence on $\mathbb{H}$. The action of $M$ on the quotient, say $\Omega$, is transitive and $\Omega e=\left\{H x_{i} \mid 1 \leq i \leq r\right\}$. The action of $G_{e}$ on this set is the desired action.

The proof of Theorem 17 depends on the construction of a surjective morphism from $e M$ to $\Omega$. To do so, we choose an element $\alpha \in \Omega e$ and use the map : $e M \rightarrow \Omega$ defined in Proposition 2, Any other choice of the element in $\Omega e$ would lead to the same construction. This is due to the following:

Proposition 18. Let $\phi: \Omega \rightarrow \Gamma$ be an injective morphism between the $M$-sets $\Omega$ and $\Gamma$, let $e$ be an idempotent in $\mathrm{I}(M)$ and let $\alpha$ be an element in $\Omega e$. Then $\operatorname{Stab}_{e M}(\alpha)=\operatorname{Stab}_{e M}(\phi(\alpha))$.

We apply the above result to a concrete example.

Example 19. For the set $5=\{1, \cdots, 5\}$, consider the following submonoid of $T_{5}$ :

$$
\begin{aligned}
M=\left\{f \in \mathrm{T}_{5} \mid f_{\mid\{1,2,3\}}\right. & \left.\in \mathrm{S}_{\{1,2,3\}},(4) f=(2) f \text { and }(5) f=(3) f\right\} \cup \\
& \left\{g \in \mathrm{T}_{5} \mid g_{\mid\{1,4,5\}} \in \mathrm{S}_{\{1,4,5\}},(4) g=(2) g \text { and }(5) g=(3) g\right\} \cup\left\{i d_{5}\right\} .
\end{aligned}
$$

Every element $f$ can be labelled as $f_{\sigma}$ with $\sigma \in \mathrm{S}_{\{1,2,3\}}$, and every $g$ can be labelled as $g_{\tau}$ with $\tau \in \mathrm{S}_{\{1,4,5\}}$, where $f_{\mid\{1,2,3\}}=\sigma$ and $g_{\mid\{1,4,5\}}=\tau$. Clearly $M$ has a transitive and faithful 
action on 5. Moreover, $\mathrm{I}(M)=M \backslash\left\{i d_{5}\right\}$, and it has two idempotents, $f_{1}$ and $g_{1}$. Note that $f_{1} M f_{1} \cong g_{1} M g_{1} \cong \mathrm{S}_{3}$. For the element $1 \in(5) f_{1}$, we have that the action of $M$ on $\Omega$ is equivalent to the action of $M$ on $f_{1} M / \equiv$, where $\equiv$ is the congruence given by

$$
\equiv=\left\{\left(m, m^{\prime}\right) \in M \mid(1) m=(1) m^{\prime}\right\} .
$$

Furthermore, if we define $N=\left[f_{1}\right]_{\equiv}=\{m \in M \mid(1) m=1\}$ and $H=N f_{1}$, we have:

$$
N=\left\{f_{1}, f_{(2,3)}, g_{1}, g_{(4,5)}\right\}, \quad H=\left\{f_{1}, f_{(2,3)}\right\} .
$$

A set of transversals of $H$ in $G_{f_{1}}$ could be $\left\{f_{1}, f_{(1,2)}, f_{(1,3)}\right\}$, thus $f_{1} M$ can be partitioned as:

$$
f_{1} M=\begin{array}{lll}
H & H f_{(1,2)} & H f_{(1,3)} \\
H g_{1} & H f_{(1,2)} g_{1} & H f_{(1,3)} g_{1}
\end{array}
$$

Note that $H$ and $H g_{1}$ lie in the same $\equiv$-class. The other 4 blocks form a $\equiv$-class on themselves. With such quotient, we recover the original action on 5.

$$
f_{1} M / \equiv=\begin{array}{|l|l|l|}
\hline H & H f_{(1,2)} & H f_{(1,3)} \\
\cline { 2 - 3 } & H f_{(1,2)} g_{1} & H f_{(1,3)} g_{1} \\
\hline
\end{array}
$$

Theorem 20. Let $M$ be a transitive transformation monoid, let $e \in \mathrm{E}(\mathrm{I}(M))$, let $\mathrm{E}(e M)=$ $\left\{e, e_{2}, \cdots, e_{n}\right\}$, and let $H$ be a subgroup of $G_{e}$. Assume that $T=\left\{x_{1}, \cdots, x_{r}\right\}$ is a transversal of $H$ in $G_{e}$ and [ $\left.\cdot\right]$ is a partition on set $\mathbb{H}=\left\{H x_{i} e_{j} \mid 1 \leq i \leq r, 1 \leq j \leq n\right\}$ satisfying Statements i) and ii) of Theorem 17 . The congruence defines a faithful and transitive action if, and only if, the following statements are satisfied.

iii) $H$ is a core-free subgroup of $G_{e}$

iv) For each pair $e_{j}, e_{j^{\prime}}$ there exists some $x_{i} \in T$ with $\left[H x_{i} e_{j}\right] \neq\left[H x_{i} e_{j^{\prime}}\right]$.

Proof. Assume that the congruence induces a faithful and transitive action. From Lemma 13 , it follows that $G_{e}$ has a transitive and faithful action on $H$. Consequently, $H$ must be a core-free subgroup of $G_{e}$ and Statement iii) holds.

Let $e_{j}$ and $e_{j^{\prime}}$ be elements of $\mathrm{E}(e M)$. Suppose that $\left[H x_{i} e_{j}\right]=\left[H x_{i} e_{j^{\prime}}\right]$, for every $1 \leq i \leq r$. Then, for every class $\left[H x_{i} e_{k}\right]$, we have that

$$
\left[H x_{i} e_{k}\right] e_{j}=\left[H x_{i} e_{j}\right]=\left[H x_{i} e_{j^{\prime}}\right]=\left[H x_{i} e_{k}\right] e_{j^{\prime}} .
$$

Since the action is faithful, we have that $e_{j}=e_{j^{\prime}}$ and Statement iv) holds.

Conversely, assume that the congruence [ $\cdot$ ] satisfies Statements iii) and iv). Let us prove that the action of $M$ on the quotient is faithful.

Let $m, m^{\prime} \in M$ be such that $\left[\left(H x_{i} e_{j}\right) m\right]=\left[\left(H x_{i} e_{j}\right) m^{\prime}\right]$ for all $x_{i} \in T, e_{j} \in \mathrm{E}(e M)$. Then $\left[H x_{i}(j) \rho_{m}\left(e_{j \bar{m}}\right)\right]=\left[H x_{i}(j) \rho_{m^{\prime}}\left(e_{j \overline{m^{\prime}}}\right)\right]$. To this regard, note that $(j) \rho_{m},(j) \rho_{m^{\prime}} \in G_{e}, e_{j} m=$ $(j) \rho_{m} e_{j \bar{m}}, e_{j} m^{\prime}=(j) \rho_{m^{\prime}} e_{j \overline{m^{\prime}}}$. Statement i) of the characterization given in Theorem 17 yields $H x_{i}(j) \rho_{m}=H x_{i}(j) \rho_{m^{\prime}}$ for all $x_{i} \in T$. Then $\left((j) \rho_{m}\right)\left((j) \rho_{m^{\prime}}^{-1}\right) \in$ Core $_{G_{e}}=\{e\}$. Consequently, $\rho_{m}=\rho_{m^{\prime}}$.

Let $e_{j} \in \mathrm{E}(e M)$ and $x_{k} \in T$. Then there exists $x_{i} \in T$ such that $H x_{i}(j) \rho_{m}=H x_{k}$. Hence $\left[H x_{k} e_{j \bar{m}}\right]=\left[H x_{k} e_{j \bar{m}^{\prime}}\right]$. Statement ii) of the characterization given in Theorem 17 implies that $e_{j \bar{m}}=e_{j \bar{m}^{\prime}}$. Since the action of $\bar{M}$ on $\mathrm{E}(e M)$ is faithful, it follows that $\bar{m}=\bar{m}^{\prime}$. The embedding of Proposition 11 yields $m=m^{\prime}$.

Therefore $M$ acts transitively and faithfully on the quotient by [· ]. The proof of the theorem is now complete. 
Corollary 21. Let $M$ be a transitive transformation monoid. If $G_{e}$ is trivial for some idempotent e in $\mathrm{I}(M)$, then $M$ has a unique transitive and faithful action up to equivalence.

Corollary 22. Let $M$ be a transitive transformation monoid. Let e $\in \mathrm{E}(\mathrm{I}(M))$, and let $H$ be a core-free subgroup of $G_{e}$. Then $M$ has a transitive and faithful action that restricts to the action of $G_{e}$ on the right coset space of $H$ in $G_{e}$ by right multiplication.

Proof. Let $\left\{x_{1}, \cdots, x_{r}\right\}$ be a transversal of $H$ in $G_{e}$ and let $\left\{e, e_{2}, \cdots, e_{n}\right\}$ be the set of idempotents of $e M$. The action of $M$ on $\mathbb{H}$ is transitive and faithful and recovers the original action of $G_{e}$ on $\left\{H x_{i} \mid 1 \leq i \leq r\right\}$.

The induced functor introduced by Steinberg in [25] clearly corresponds with the partition described in Corollary 22. Our main goal now is to give a criterion for two $M$-actions determined in Theorem 20 to be equivalent. In the following, we assume that $M$ is a monoid satisfying the statements of Theorem 20 .

Proposition 23. Let [·] be a equivalence relation on the set $\mathbb{H}$ satisfying the statements of Theorem 20. Then $\operatorname{Stab}_{e M}([H])=[H]$ and $\operatorname{Stab}_{G_{e}}([H])=[H] \cap G_{e}=H$. Moreover, $\operatorname{Stab}_{e M}\left(\left[H x_{i}\right]\right) e=\operatorname{Stab}_{G_{e}}\left(\left[H x_{i}\right]\right)=H^{x_{i}}$, for $1 \leq i \leq r$.

Proposition 24. Let $[\cdot]_{1},[\cdot]_{2}$ be two equivalence relations on the set $\mathbb{H}$ satisfying the statements of Theorem 20. Let $\phi:[\cdot]_{1} \rightarrow[\cdot]_{2}$ be a bijective morphism of $M$-sets. Then $\phi\left([H]_{1}\right)=\left[H x_{i}\right]_{2}$ for some transversal $x_{i}$ of $H$ in $G_{e}$.

Proof. Assume that $\phi\left([H]_{1}\right)=\left[H x_{i} e_{j}\right]_{2}$ for some idempotent $e_{j} \in e M$. As $[H]_{1} e=[H]_{1}$, then $\left[H x_{i} e_{j}\right]_{2}=\left[H x_{i}\right]_{2}$.

Proposition 25. Let $[\cdot]_{1},[\cdot]_{2}$ be two equivalence relations on the set $\mathbb{H}$ satisfying the statements of Theorems 17 and 20. Let $\phi:[\cdot]_{1} \rightarrow[\cdot]_{2}$ be a bijective morphism of $M$-sets with $\phi\left([H]_{1}\right)=\left[H x_{i}\right]_{2}$, then $x_{i} \in \mathrm{N}_{G_{e}}(H)$.

Proof. Applying Propositions 18 and 23, we have $[H]_{1}=\operatorname{Stab}_{e M}\left([H]_{1}\right)=\operatorname{Stab}_{e M}\left(\left[H x_{i}\right]_{2}\right)$. Thus $H=[H]_{1} e=\operatorname{Stab}_{e M}\left(\left[H x_{i}\right]_{2}\right) e$. By Proposition 23 on Stabilizers, $\operatorname{Stab}_{e M}\left(\left[H x_{i}\right]_{2}\right) e=$ $x_{i}^{-1} \operatorname{Stab}_{G_{e}}\left([H]_{2}\right) x_{i}$ and $\operatorname{Stab}_{e M}\left([H]_{2}\right) e=H$. Consequently, $x_{i} \in \mathrm{N}_{G_{e}}(H)$.

We close our treatment of transitive transformation monoids with an example showing how we can use Theorem 20 to construct non-equivalent transitive and faithful actions of $M$ that restrict to a given transitive and faithful action of $G_{e}$.

Example 26. Let $G=\mathrm{S}_{3}$ be the symmetric group of order 3. We will denote the identity in $G$ by $e$. Let $2=\{1,2\}$ be a set with two elements and let $N$ be the submonoid of $\mathrm{T}_{2}$ containing the identity and the two constant functions, that is $N=\left\{1_{2}, \varsigma_{1}, \varsigma_{2}\right\}$. Consider the following submonoid of the wreath product:

$$
M=\left\{\left(\psi_{g}, \varsigma_{j}\right) \mid g \in G, j \in 2\right\} \cup\left\{\left(\varphi_{e}, 1_{2}\right)\right\} \leq G \imath_{2} N .
$$

In this example, $\mathrm{I}(M)=\left\{\left(\varphi_{g}, \varsigma_{j}\right) \mid g \in G, j \in 2\right\}$ and $\mathrm{E}(\mathrm{I}(M))=\left\{\widehat{e}_{1}=\left(\psi_{e}, \varsigma_{1}\right), \widehat{e}_{2}=\right.$ $\left.\left(\psi_{e}, \varsigma_{2}\right)\right\}=\mathrm{E}\left(\widehat{e}_{1} M\right)$. The maximal subgroup of $M$ at $\widehat{e}_{1}$ is isomorphic to $G$. Let $H=$ $\left\{\widehat{e}_{1},\left(\psi_{(2,3)}, \varsigma_{1}\right)\right\} \leq G_{\widehat{e}_{1}}$, and let $\left\{x_{1}=\widehat{e}_{1}, x_{2}=\left(\psi_{(1,2)}, \varsigma_{1}\right), x_{3}=\left(\psi_{(1,3)}, \varsigma_{1}\right)\right\}$ be a right transversal of $H$ in $G_{\widehat{e}_{1}}$. In this case, all the relations $\simeq^{i}$ for $i=1,2,3$ are total. The following seven equivalence relations on the set $\mathbb{H}=\left\{H x_{i} e_{j} \mid 1 \leq i \leq 3,1 \leq j \leq n\right\}$ satisfy the statements of Theorem 20 . 


\begin{tabular}{|l|l|l|}
\hline$H$ & $H x_{2}$ & $H x_{3}$ \\
\hline$H \widehat{e}_{2}$ & $H x_{2} \widehat{e}_{2}$ & $H x_{3} \widehat{e}_{2}$ \\
\hline
\end{tabular}

\begin{tabular}{|l|l|l|}
\hline$H$ & $H x_{2}$ & $H x_{3}$ \\
\cline { 2 - 3 }$H \widehat{e}_{2}$ & $H x_{2} \widehat{e}_{2}$ & $H x_{3} \widehat{e}_{2}$ \\
\hline
\end{tabular}

\begin{tabular}{|l|l|l|}
\hline$H$ & $H x_{2}$ & $H x_{3}$ \\
\cline { 1 - 1 }$H \widehat{e}_{2}$ & $H x_{2} \widehat{e}_{2}$ & $H x_{3} \widehat{e}_{2}$ \\
\hline
\end{tabular}

\begin{tabular}{|l|l|l|}
\hline$H$ & $H x_{2}$ & $H x_{3}$ \\
\cline { 1 - 2 }$H \widehat{e}_{2}$ & $H x_{2} \widehat{e}_{2}$ & $H x_{3} \widehat{e}_{2}$ \\
\hline
\end{tabular}

\begin{tabular}{|l|l|l|}
\hline$H$ & $H x_{2}$ & $H x_{3}$ \\
\cline { 3 - 3 }$H \widehat{e}_{2}$ & $H x_{2} \widehat{e}_{2}$ & $H x_{3} \widehat{e}_{2}$ \\
\hline
\end{tabular}

\begin{tabular}{|l|l|l|}
\hline$H$ & $H x_{2}$ & $H x_{3}$ \\
$H \hat{e}_{2}$ & $H x_{2} \widehat{e}_{2}$ & $H x_{3} \widehat{e}_{2}$ \\
\hline
\end{tabular}

\begin{tabular}{|l|l|l|}
\hline$H$ & $H x_{2}$ & $H x_{3}$ \\
\cline { 1 - 1 }$H \widehat{e}_{2}$ & $H x_{2} \widehat{e}_{2}$ & $H x_{3} \widehat{e}_{2}$ \\
\hline
\end{tabular}

The partition on the top corresponds to the induced action on $H$. Applying Theorem 20. we have that $M$ has a transitive and faithful action on each of the seven quotient sets, and each action restricts to the action of $G_{\widehat{e}_{1}}$ on $H$ by right multiplication. Recall that not every quotient of the induced action satisfies this property (consider the extreme case when every coset $H x_{i} e_{j}$ lies in the same [·]-class). Since $\mathrm{N}_{G_{\widehat{e}_{1}}}(H)=H$, we can apply Proposition 25 to conclude that these actions are not equivalent. Moreover, in this particular case, $M$ is isomorphic to submonoid of all three transformations monoids $T_{4}, T_{5}$, and $T_{6}$.

\section{Primitive monoid actions}

Following [25], an action of a monoid $M$ on a set $\Omega$ is said to be primitive if it admits no nontrivial proper congruences. This is to say that if the action is primitive, then every congruence on $\Omega$ must be equal to the diagonal relation, denoted by $\Delta_{\Omega}=\{(\alpha, \beta) \in \Omega \times \Omega \mid \alpha=\beta\}$, or to the total relation, denoted by $\nabla_{\Omega}=\Omega \times \Omega$.

Primitive groups actions are completely characterised in terms of core-free maximal subgroups. We reproduce here this well-known and important result.

Theorem 27 ([3, Theorem 1.1.5]). Let $G$ be a group. The following statements are equivalent:

i) $G$ is a primitive permutation group.

ii) There exists a core-free maximal subgroup of $G$.

The main aim of this section is to describe and characterise primitive transitive transformation monoids. If $|\Omega| \leq 2$, every action on $\Omega$ is primitive, so we shall assume in the sequel that $|\Omega| \geq 3$. In general, primitive actions are not transitive as the following example shows.

Example 28. Consider the submonoid $M$ of $\mathrm{T}_{3}$ generated by the transformations $[1,1,2]$, and $[1,3,1]$. The monoid $M$ contains 6 elements and its minimal ideal is given by $\mathrm{I}(M)=\{[1,1,1]\}$. The action of $M$ on 3 is primitive as any congruence containing a pair of different elements is equal to the total relation. However, this action is not transitive as $2 M=3 M=3$, but $1 M=\{1\}$.

Throughout this section, $M$ will denote a monoid having a transitive and faithful action. Let $e \in \mathrm{E}(\mathrm{I}(M))$. Write $\mathrm{E}(e M)=\left\{e_{1}, e_{2}, \cdots e_{n}\right\}$. Let $H$ be a subgroup of $G_{e}=e M e$. If $T=\left\{e=x_{1}, \cdots, x_{r}\right\}$ is a right transversal of $H$ in $G_{e}$, let $\mathbb{H}=\left\{H x_{i} e_{j} \mid 1 \leq i \leq r, 1 \leq j \leq n\right\}$ be the associated partition of $e M$.

Theorem 29. Assume that [ $[$ ] is a congruence on the $M$-set $\mathbb{H}$ satisfying the statements of Theorem 17. If $H \neq G_{e}$, and the action of $M$ on $[\cdot]$ is primitive, then $\left[H x_{i} e_{j}\right]=\left[H x_{i} e_{j^{\prime}}\right]$ if and only if $j \simeq^{i} j^{\prime}$, for all $i \in\{1, \cdots, r\}$. 
Proof. Let $i \in\{1, \cdots, r\}$. Only the sufficiency condition is in doubt. Consider the following equivalence relation $\simeq$ on the set $\left\{H x_{i} e_{j}\right\}$ :

$$
H x_{i} e_{j} \simeq H x_{i^{\prime}} e_{j^{\prime}} \text { if and only if } i=i^{\prime} \text { and } j \simeq^{i} j^{\prime} .
$$

By Theorem 20, the equivalence relation associated to the partition [ · ] is contained in $\simeq$. Therefore $\simeq$ induces an equivalence relation on the partition $[\cdot]$. We show that $\simeq$ is a congruence. Let $H x_{i} e_{j} \simeq H x_{i} e_{j^{\prime}}$ and $m \in M$. Then:

$$
\begin{aligned}
& \left(H x_{i} e_{j}\right) m=H x_{k}\left(e_{j \bar{m}}\right) \text { with } x_{i}(j) \rho_{m} \in H x_{k} \\
& \left(H x_{i} e_{j^{\prime}}\right) m=H x_{k}\left(e_{j^{\prime} \bar{m}}\right) \text { with } x_{i}\left(j^{\prime}\right) \rho_{m} \in H x_{k} .
\end{aligned}
$$

Let $m^{\prime} \in M$. According to the proof of Proposition 11 , it follows the equation $(j) \rho_{m}(j \bar{m}) \rho_{m^{\prime}}=$ $(j) \rho_{m m^{\prime}}$. Hence

$$
\begin{aligned}
H x_{k}(j \bar{m}) \rho_{m^{\prime}} & =H x_{i}(j) \rho_{m}(j \bar{m}) \rho_{m^{\prime}}=H x_{i}(j) \rho_{m m^{\prime}} \\
& =H x_{i}\left(j^{\prime}\right) \rho_{m m^{\prime}}=H x_{i}\left(j^{\prime}\right) \rho_{m}\left(j^{\prime} \bar{m}\right) \rho_{m^{\prime}} \\
& =H x_{k}(j \bar{m}) \rho_{m^{\prime}} .
\end{aligned}
$$

Therefore $\left(H x_{i} e_{j}\right) m \simeq\left(H x_{i} e_{j^{\prime}}\right) m$. Then $\simeq$ is a congruence on the primitive $M$-set [ ] . Moreover, $|T|=\left|G_{e}: H\right|=|\Omega e| \geq 2$. Hence there exist $x_{i} \neq x_{i^{\prime}} \in T$ such that $\left[H x_{i} e\right]$ and $\left[H x_{i^{\prime}} e\right]$ are not $\simeq$-related. By primitivity, $\simeq$ coincides with the diagonal relation on partition $[\cdot]$. Hence $j \simeq^{i} j^{\prime}$ implies $\left[H x_{i} e_{j}\right]=\left[H x_{i} e_{j^{\prime}}\right]$

We derive now some interesting consequences of Theorem 29.

Corollary 30. Two primitive and transitive actions of $M$ satisfying that the restriction of the action of $M$ to eMe is equivalent to the action of $G_{e}$ by right multiplication on the coset space of $H$ are equivalent.

Corollary 31. Suppose that the action of $M$ on $\Omega$ is transitive, primitive and faithful, and $|\Omega e| \geq 2$. Then if $e_{j} \neq e_{j^{\prime}} \in e M$, there exists $x_{i} \in T$ such that $j \not^{i} j^{\prime}$.

Theorem 32. Assume that the action of $M$ on $\Omega$ is faithful, transitive and primitive and $|\Omega e| \geq 2$. Let $[\cdot]$ be a congruence on $\mathbb{H}$ defining this action and satisfying that the restriction to $G_{e}$ on $\Omega e$ is equivalent to the action of $G_{e}$ on the set of right cosets of $H$. Then, for all $e_{j} \neq e_{j^{\prime}} \in e M$, there exist finite sequences $\left(x_{i_{t}}\right)_{t=1}^{s}$ and $\left(e_{j_{t}}\right)_{t=1}^{s+1}$ of elements in $T$ and $\mathrm{E}(e M)$, respectively, such that

$$
j=j_{1} \simeq^{i_{1}} j_{2} \simeq^{i_{2}} \ldots \simeq^{i_{s-1}} j_{s} \simeq^{i_{s}} j_{s+1}=j^{\prime}
$$

Proof. We consider the following binary relation $\bumpeq$ on $\mathbb{H}$ :

$$
H x_{i} e_{j} \bumpeq H x_{i^{\prime}} e_{j^{\prime}} \quad \text { if and only if } \exists k \in\{1, \cdots, r\} \text { with } j \simeq^{k} j^{\prime}
$$

If $\left[H x_{i} e_{j}\right]=\left[H x_{i} e_{j^{\prime}}\right]$, then $j \simeq^{i} j^{\prime}$. This means that $[\cdot]$ is contained in $\bumpeq$ and so $\bumpeq$ induces a binary relation on $[\cdot]$.

Let $\approx$ be the transitive closure of $\bumpeq$. Thus, $\approx$ is a binary equivalence relation on $[\cdot]$. We prove that $\approx$ is a congruence. Let $m \in M$ and suppose that $\left[H x_{i} e_{j}\right] \bumpeq\left[H x_{i^{\prime}} e_{j^{\prime}}\right]$. Then there exists some $k \in\{1, \cdots, r\}$ with $j \simeq^{k} j^{\prime}$. By Theorem 20, we have $\left[H x_{l}\left(e_{j \bar{m}}\right)\right]=\left[H x_{l}\left(e_{\left.j^{\prime} \bar{m}\right)}\right]\right.$ 
with $x_{k} \rho_{m}(j) \in H x_{l}$. Hence $j m \simeq^{l} j^{\prime} m$ and $\left[\left(H x_{i} e_{j}\right)\right] m=\left[\left(H x_{i} e_{j}\right) m\right] \bumpeq\left[\left(H x_{i^{\prime}} e_{j^{\prime}}\right) m\right]=$ $\left[\left(H x_{i^{\prime}} e_{j^{\prime}}\right)\right] m$.

The primitivity of $M$ implies that either $\approx$ is the diagonal relation or $\approx$ is the total relation. Moreover, $|T|=\left|G_{e}: H\right|=|\Omega e| \geq 2$. Therefore, there exists $x \in T$ such that $x \notin H$. Then $[H]$ and $[H x]$ are two different $\bumpeq$ - related elements of $[\cdot]$. Consequently, $\approx$ is the total relation on [ $\cdot]$. Thus, for any pair of idempotents $e_{j}, e_{j^{\prime}}$, there exist finite sequences $\left(x_{i_{t}}\right)_{t=1}^{s}$ of $T$ and $\left(e_{j_{t}}\right)_{t=1}^{s+1}$ of $e M$ such that $j_{1}=j, j_{s+1}=j^{\prime}$ and $j_{t} \simeq^{i_{t}} j_{t+1}$ for all $t=1, \cdots, s$.

Theorem 32 can be better understood as follows: let $\Gamma=(E, D)$ be a graph whose set of vertices is the set of idempotents in $e M$. Two idempotents $e_{j}$ and $e_{j}^{\prime}$ are connected by an edge in $D$ if there exists some transversal $x_{i} \in T$ for which $j \simeq^{i} j^{\prime}$ holds. Theorem 32 implies that $\Gamma$ is a connected graph.

One of the first consequences of the last theorem is that in a monoid $M$ with more than one idempotent in $e M$, no induced action, in the sense of [25], can be primitive.

Corollary 33. If $|\mathrm{E}(e M)| \geq 2$, for any subgroup $H$ in $G_{e}$ the corresponding induced action cannot be primitive.

Proof. We cannot find a sequence as in Theorem 32 relating $\mathrm{He}$ and $\mathrm{He}_{2}$, for different idempotents $e$ and $e_{2}$.

We bring the first part of this section to a close with a nice consequence of Theorem 32 . It reveals another strikking difference between group actions and monoids actions.

Corollary 34. Let $M$ be a transitive transformation monoid whose maximal subgroup $G_{e}$ is a non-trivial nilpotent group, for some idempotent e in $\mathrm{I}(M)$. Then $M$ has a primitive action if and only if $M$ is a cyclic group of order prime.

Proof. If $M$ is a cyclic group of order prime then $M$ is primitive by Theorem 27. Thus, it has a primitive action. On the converse, assume that $M$ has a primitive action. Assume towards a contradiction that $\mathrm{E}(e M)$ contains more than one idempotent, that is $\mathrm{E}(e M)=$ $\left\{e, e_{2}, \cdots, e_{n}\right\}$. Since $G_{e}$ is a non-trivial nilpotent group, the subgroup $H$ must be equal to $\{e\}$ and so $|\Omega e|=\left|G_{e}\right|=r \geq 2$. By Corollary 31, the action of $M$ on $\Omega$ is necessarily equivalent to

$$
e M=\begin{array}{|c|c|c|c|}
\hline e & x_{2} & \cdots & x_{r} \\
\hline e_{2} & x_{2} e_{2} & \cdots & x_{r} e_{2} \\
\hline \vdots & \vdots & \ddots & \vdots \\
\hline e_{n} & x_{2} e_{n} & \cdots & x_{r} e_{n} \\
\hline
\end{array}
$$

This configuration contradicts Theorem 32 . Therefore, $\mathrm{E}(e M)$ contains a unique idempotent which must be equal to $e$. It follows that $e M$ is a group and by Corollary 8 , the monoid $M$ is equal to $G_{e}$. Thus, $M$ is a primitive non-trivial nilpotent group. By [9, Chapter A, Theorem 8.3] $M$ is a cyclic group of prime order.

Corollary 35. If $|\Omega e|=2$ for some idempotent e in $\mathrm{I}(M)$, then the action of $M$ on $\Omega$ cannot be primitive. 
Proof. Note that $\left|G_{e}: H\right|=|\Omega e|=2$. Then $H$ is a normal subgroup of $G_{e}$ and so $H=\{e\}$. In particular, $G_{e}$ is a cyclic group of order 2 . Assume towards a contradiction that the action on $\Omega$ is primitive then, by Corollary 34 , the monoid $M$ is equal to the cyclic group of order 2 . Moreover, for $e \in \mathrm{I}(M)$, we have $e M=M=G_{e}$. Consequently, the action on $\Omega$ is equivalent to $e M /[\cdot]$ for some congruence $[\cdot]$. Note that $|\Omega| \geq 3$, whereas $|e M /[\cdot]| \leq 2$. Therefore, the action cannot be primitive.

Having identified some of the natural properties of the primitive transitive transformation monoids, we are led naturally to inquire about characterisation results. Having seen that transitive permutation groups play an important role in the study of transitive permutation monoids, it is reasonable to think that primitive permutation groups play a similar role in the study of primitive transitive transformation monoids. The first indication that this is true follows from the following lemma.

Lemma 36 ([25, Proposition 5.2]). If the action of $M$ on $\Omega$ is primitive and e is an idempotent on $M$, then the action of eMe on $\Omega e$ is primitive.

Of course, the interesting case is when $e \in \mathrm{I}(M)$. In this case, $H$ is a core-free maximal subgroup of $G_{e}$, that is, $G_{e}$ is a primitive permutation group. The converse of Lemma 36 does not hold as the following example shows.

Example 37. Let $G$ be a group having a maximal core-free subgroup $H$. Let $e$ be the identity of $G$. By Theorem 27 the action of $G$ on $\Theta=\{H g \mid g \in G\}$ by right multiplication is primitive. For a natural number $n$, consider the monoid $M$ we constructed in Example 7 from the cartesian product $G \times \mathrm{n}$. The monoid $M$ acts on the set $\Omega=\Theta \times \mathrm{n}$ by $(H a, i)(b, j)=$ $(H a b, \min (i, j))$. The restriction of this action to $G=G_{(e, 1)}$ is primitive as it is equivalent to the action of $G$ on $\Theta$. However, for $n \geq 2$ the set $\Omega$ admits a non-trivial congruence $\backsim$ given by $(H g, i) \backsim\left(H g^{\prime}, i^{\prime}\right)$ if and only if $H g=H g^{\prime}$.

In the first part of this section, we have established some results which, when taken together, give a rather good picture of the structure of a primitive transitive transformation monoids in which $|\Omega e| \geq 2$. If $|\Omega e|=2$, the action cannot be primitive. Hence, we must consider two different cases: $|\Omega e| \geq 3$ and $|\Omega e|=1$.

\subsubsection{Case $1-|\Omega e| \geq 3$}

Theorem 38. Assume that $M$ has a faithful and transitive action on $\Omega$ for which the restriction of $G_{e}$ on $\Omega e$ is equivalent to the action of $G_{e}$ on the set of right cosets of $H$. Let $[\cdot]$ be the congruence on $\mathbb{H}$ defining this action. Assume that $|\Omega e| \geq 3$. Then the action of $M$ on $\Omega$ is primitive if and only if the following statements hold:

i) $H$ is a core-free maximal subgroup of $G_{e}$;

ii) $\left[H x_{i} e_{j}\right]=\left[H x_{i} e_{j^{\prime}}\right]$ if and only if $j \simeq^{i} j^{\prime}$ for all $i \in\{1, \cdots, r\}$;

iii) for all $e_{j} \neq e_{j^{\prime}} \in e M$, there exist finite sequences $\left(x_{i_{t}}\right)_{t=1}^{s}$ and $\left(e_{j_{t}}\right)_{t=1}^{s+1}$ of elements $T$ and $\mathrm{E}(e M)$, respectively, such that

$$
j=j_{1} \simeq^{i_{1}} j_{2} \simeq^{i_{2}} \ldots \simeq^{i_{s-1}} j_{s} \simeq^{i_{s}} j_{s+1}=j^{\prime}
$$


Proof. Keeping in mind the previous results, it is clear that only the sufficiency of the conditions is in doubt. Assume that $M$ has a faithful and transitive action on the quotient by [ · ] and satisfies Statements i) to iii). We prove that this action is primitive.

Suppose there exists a proper congruence $\doteqdot$ relating two distinct classes $\left[H x_{i_{0}} e_{j_{0}}\right]$ and $\left[H x_{i_{0}^{\prime}} e_{j_{0}^{\prime}}\right]$. Hence the classes $\left[H x_{i_{0}}\right] e=\left[H x_{i_{0}}\right]$ and $\left[H x_{i_{0}^{\prime}}\right] e=\left[H x_{i_{0}^{\prime}}\right]$ are $\doteqdot$-related.

Assume that $i_{0}=i_{0}^{\prime}$. Since $\left[H x_{i_{0}} e_{j_{0}}\right]$ and $\left[H x_{i_{0}} e_{j_{0}^{\prime}}\right]$ are distinct classes, there exists an element $m \in M$ with $H x_{k_{0}}=H x_{i_{0}}\left(j_{0}\right) \rho_{m} \neq H x_{i_{0}}\left(j_{0}^{\prime}\right) \rho_{m}=H x_{k_{0}^{\prime}}$. Again, as $\doteqdot$ is a congruence relating $\left[H x_{i_{0}} e_{j_{0}}\right]$ and $\left[H x_{i_{0}} e_{j_{0}^{\prime}}\right]$, multiplying by $m e$, we conclude that $\left[H x_{k_{0}}\right]$ and $\left[H x_{k_{0}^{\prime}}\right]$ are $\doteqdot$-related.

In both cases, we conclude that a pair of classes $\left[H x_{k_{0}}\right]$ and $\left[H x_{k_{0}^{\prime}}\right]$ are $\doteqdot$-related for some pair of different elements $x_{k_{0}}$ and $x_{k_{0}^{\prime}}$ of $T$.

Thus, the action of $M$ restricts to the action of $G_{e}$ on the coset space of $H$ in $G$ by right multiplication. This action is primitive because $H$ is a core-free maximal subgroup of $G$. Hence $\left[H x_{i}\right] \doteqdot\left[H x_{i^{\prime}}\right]$ for all $i, i^{\prime} \in\{1, \cdots, r\}$. We conclude $\left[H x_{i} e_{j}\right] \doteqdot\left[H x_{i^{\prime}} e_{j}\right]$ for all $i, i^{\prime} \in\{1, \cdots, r\}$ and $1 \leq j \leq n$.

Let $\left[H x_{i} e_{j}\right]$ and $\left[H x_{i^{\prime}} e_{j^{\prime}}\right]$ be two arbitrary classes with $j \neq j^{\prime}$, then there exists a finite sequence of elements $\left(x_{i_{t}}\right)_{t=1}^{s}$ of $T$ and a finite sequence of idempotents $\left(e_{j_{t}}\right)_{t=1}^{s+1}$ of $e M$ satisfying $j=j_{1} \simeq^{i_{1}} j_{2} \simeq^{i_{2}} \ldots \simeq \simeq^{i_{s-1}} j_{n} \simeq^{i_{s}} j_{s+1}=j^{\prime}$. The condition $j_{t} \simeq^{i_{t}} j_{t+1}$ implies $\left[H x_{i_{t}} e_{j_{t}}\right]=$ $\left[H x_{i_{t}} e_{j_{t+1}}\right]$ for all $t=1, \cdots s$. Hence $\left[H x_{i} e_{j_{t}}\right] \doteqdot\left[H x_{i_{t}} e_{j_{t}}\right]=\left[H x_{i_{t}} e_{j_{t+1}}\right] \doteqdot\left[H x_{i^{\prime}} e_{j_{t+1}}\right]$. The existence of the above chain implies that $\left[H x_{i} e_{j}\right]$ and $\left[H x_{i^{\prime}} e_{j^{\prime}}\right]$ are $\doteqdot$-related. Thus, $\doteqdot$ is necessarily the total relation. The proof of the theorem is now complete.

Theorem 39. Let $M$ be a transitive transformation monoid such that $G_{e}$ is non-trivial. Then $M$ has a faithful primitive transitive action on a set $\Omega$ if and only if there exists a maximal core-free subgroup $H$ of $G_{e}$ such that the relations $\simeq$ defined by $H$ on the set of idempotents of eM satisfy the following statements:

i) for all $e_{j} \neq e_{j^{\prime}} \in e M$, there exist finite sequences $\left(x_{i_{t}}\right)_{t=1}^{s}$ and $\left(e_{j_{t}}\right)_{t=1}^{s+1}$ of elements of $T$ and $\mathrm{E}(e M)$, respectively, such that

$$
j=j_{1} \simeq^{i_{1}} j_{2} \simeq^{i_{2}} \ldots \simeq^{i_{s-1}} j_{s} \simeq^{i_{s}} j_{s+1}=j^{\prime}
$$

ii) for all $e_{j} \neq e_{j^{\prime}} \in e M$, there exists $x_{i} \in T$ with $j \not^{i} j^{\prime}$.

Proof. If there is a faithful and primitive action, the above statements follow immediately from Theorem 32 and Corollary 31 .

Conversely, assume that $M$ is a transitive transformation monoid such that $G_{e}$ has a corefree maximal subgroup $H$ satisfying the statements of the theorem. Let [ $\cdot]$ be the equivalence relation in $\Lambda$ :

$$
\left[H x_{i} e_{j}\right]=\left[H x_{i} e_{j^{\prime}}\right] \quad \text { if and only if } \quad j \simeq^{i} j^{\prime}
$$

It is clear that $[\cdot]$ satisfies Statement iii) of Theorem 20. We shall see that it also satisfies Statement iv). To this end, let $m \in M$ and let $\left[H x_{i} e_{j}\right]=\left[H x_{i} e_{j^{\prime}}\right]$. It holds

$$
H x_{i} e_{j} m=H x_{i}(j) \rho_{m}\left(e_{j \bar{m}}\right), \quad \text { and } \quad H x_{i} e_{j^{\prime}} m=H x_{i}\left(j^{\prime}\right) \rho_{m}\left(e_{j^{\prime} \bar{m}}\right)
$$


As $j \simeq^{i} j^{\prime}$, we have $H x_{i} \rho_{m}(j)=H x_{i} \rho_{m}\left(j^{\prime}\right)=H x_{k}$. We need to check that $j \bar{m} \simeq^{k} j^{\prime} \bar{m}$. Let $m^{\prime}$ be an element in $M$, it holds

$$
\begin{aligned}
H x_{k}(j \bar{m}) \rho_{m^{\prime}} & =H x_{i}(j) \rho_{m}(j \bar{m}) \rho_{m^{\prime}}=H x_{i}(j) \rho_{m m^{\prime}}= \\
& =H x_{i}\left(j^{\prime}\right) \rho_{m m^{\prime}}=H x_{i}\left(j^{\prime}\right) \rho_{m}\left(j^{\prime} \bar{m}\right) \rho_{m^{\prime}}=H x_{k}\left(j^{\prime} \bar{m}\right) \rho_{m^{\prime}}\left(j^{\prime} \bar{m}\right)
\end{aligned}
$$

It follows from Theorem 20 that $M$ has a transitive and faithful action on $[\cdot]$. Moreover, this action satisfies conditions of Theorem 38. Consequently, $M$ has a primitive, transitive and faithful action on $[\cdot]$.

\section{Constructing primitive actions}

In this subsection we provide a method to construct primitive transformation monoids by means of primitive permutation groups. The reader should note that the wreath product construction studied in subsection 1.3 is an effective one. We will follow the notation introduced there.

Theorem 40. Let $G$ be a primitive permutation group. For any natural number $n$ and any submonoid $N$ of $\mathrm{T}_{\mathrm{n}}$ containing all the constant mappings, there exists a submonoid $M$ of $G \ln _{\mathrm{n}} N$ such that $M$ has a primitive, transitive and faithful action, the maximal subgroups at every idempotent in the minimal ideal of $M$ are isomorphic to $G$, and the restriction of the action of $M$ to these maximal subgroups coincides with the primitive action of $G$.

Proof. Let $H$ be a core-free maximal subgroup of $G$. Let us denote the identity element in $G$ by $e$. Let $n$ be a natural number and let $N$ be a submonoid of the full transformation monoid $\mathrm{T}_{\mathrm{n}}$ containing the set $\mathrm{I}\left(\mathrm{T}_{\mathrm{n}}\right)$ of all constant mappings on $\mathrm{n}$. In this case, $\mathrm{I}(N)=$ $\mathrm{I}\left(\mathrm{T}_{\mathrm{n}}\right)=\left\{\varsigma_{j} \mid j \in \mathrm{n}\right\}$. As in subsection 1.3 , we consider the subsemigroup $L$ of $G \mathrm{n}_{\mathrm{n}} N$ given by $L=\left\{\left(\psi_{g}, \varsigma_{j}\right) \mid g \in G, j \in \mathrm{n}\right\}$. Finally, consider the set

$$
S=\left\{\left(\psi, \varsigma_{j}\right) \mid \text { there exists } g \in G \text { such that for all } k \in \mathrm{n},(k) \psi \in H g, j \in \mathrm{n}\right\} .
$$

We claim that $S$ is a subsemigroup of $G 2_{n} N$. In fact, let $\left(\psi, \varsigma_{j}\right)$ and $\left(\psi^{\prime}, \varsigma_{j^{\prime}}\right)$ be two elements in $S$. Let $g$ and $g^{\prime}$ be the elements in $G$ satisfying that, for all $k \in \mathrm{n}$, the equations $(k) \psi \in H g$ and $(k) \psi^{\prime} \in H g^{\prime}$ hold. The product of $\left(\psi, \varsigma_{j}\right)$ and $\left(\psi^{\prime}, \varsigma_{j^{\prime}}\right)$ in $S$ is given by

$$
\left(\psi, \varsigma_{j}\right)\left(\psi^{\prime}, \varsigma_{j^{\prime}}\right)=\left(\varphi, \varsigma_{j^{\prime}}\right), \quad \text { where }(k) \varphi=(k) \psi\left(k \varsigma_{j}\right) \psi^{\prime} \quad \text { for all } k \in \mathrm{n} .
$$

Note that $k \varsigma_{j}=j$ and $(k) \varphi=(k) \psi(j) \psi^{\prime}$. Moreover, $(j) \psi^{\prime}=h g^{\prime}$ for some $h \in H$. Hence, for all $k \in \mathrm{n}$, we have

$$
(k) \varphi=(k) \psi\left(k \varsigma_{j}\right) \psi^{\prime} \in H g h g^{\prime} .
$$

Let $M=S^{1}$. Clearly, $M$ is a submonoid of $G l_{\mathrm{n}} N$ containing the subsemigroup $L$. By Theorem 12, $M$ has a transitive and faithful action on $L$ by right multiplication and $G$ is isomorphic to the maximal subgroup of $M$ at each idempotent of $\mathrm{I}(M)$. Clearly, the restriction of the action of $M$ to $G$ is just the action of $G$ on the right coset space of $H$ in $G$ by right multiplication. Consider the $|G: H|$ equivalence relations $\simeq^{i}$ defined on $\mathrm{n}=\{1, \cdots, n\}$. By Proposition 16, we have that $k \simeq^{1} k^{\prime}$ if and only if $H(k) \psi=H\left(k^{\prime}\right) \psi$, for all $\left(\psi, \varsigma_{j}\right)$ in $M$. Hence $\simeq^{1}$ is the total relation on $\mathrm{n}$ and it fulfils the condition stated in Theorem 32 . Let us check if it also satisfies Statement ii) in Theorem 39. Let $h \neq e$ be an element in $H$, 
there exists a coset $H x_{i}$ such that $h \notin H^{x_{i}}$, as $\operatorname{Core}_{G}(H)=\{e\}$. Hence, $H x_{i} h \neq H x_{i}$. Let $k, k^{\prime}$ be two arbitrary elements in $\mathrm{n}$ and let $\left(\psi, \varsigma_{j}\right)$ be an element in $M$ such that $(k) \psi=e$ and $\left(k^{\prime}\right) \psi=h$. As $H x_{i}(k) \psi \neq H x_{i}\left(k^{\prime}\right) \psi$, then $k \not^{i} k^{\prime}$. By Theorem 39, $M$ is a primitive transformation monoid.

We close our treatment of primitive monoids with an example showing how can we discriminate which actions of a transitive transformation monoid are primitive.

Example 41. Let $G=\mathrm{S}_{3}$ be the symmetric group of order 3. We will denote the identity in $G$ by $e$. Let $H$ be any subgroup of $G$ of order 2 , hence $|G: H|=3$. Let $2=\{1,2\}$ be a set with two elements and let $N$ be the submonoid of $\mathrm{T}_{2}$ containing the identity and the two constant mappings, that is $N=\left\{1_{2}, \varsigma_{1}, \varsigma_{2}\right\}$. Consider the following submonoids of the wreath

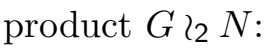

$$
\begin{aligned}
& L=\left\{\left(\psi_{g}, \varsigma_{j}\right) \mid g \in G, j \in 2\right\} \\
& S=\left\{\left(\psi, \varsigma_{j}\right) \mid \text { there exists } g \in G \text { such that for all } k \in 2,(k) \psi \in H g, j \in 2\right\} .
\end{aligned}
$$

The monoid $M=S^{1}$ contains $L$. By Theorem $12, M$ has a transitive and faithful action on $L$. In fact, for the idempotent $\widehat{e}_{1}=\left(\varphi_{e}, \varsigma_{1}\right)$, we have $\widehat{e}_{1} M=L$. Therefore $\widehat{e}_{1} M$ has only two idempotents, $\widehat{e}_{1}$ and $\widehat{e}_{2}=\left(\psi_{e}, \varsigma_{2}\right)$. The maximal subgroup of $M$ at $\widehat{e}_{1}$ is isomorphic to $G$. Let $\left\{x_{1}=\widehat{e}_{1}, x_{2}, x_{3}\right\}$ be a right transversal of $H$ in $G_{\widehat{e}_{1}}$. In this case, $\simeq^{1}$ is the total relation, whereas $\simeq^{2}$ and $\simeq^{3}$ are equal to the diagonal relation. The following two equivalence relations on the set $\mathbb{H}=\left\{H x_{i} \widehat{e}_{j} \mid 1 \leq i \leq 3,1 \leq j \leq 2\right\}$ satisfy statements of Theorem 20 .

\begin{tabular}{|l|l|l|}
\hline$H$ & $H x_{2}$ & $H x_{3}$ \\
\hline$H \widehat{e}_{2}$ & $H x_{2} \widehat{e}_{2}$ & $H x_{3} \widehat{e}_{2}$ \\
\hline
\end{tabular}

\begin{tabular}{|l|l|l|}
\hline$H$ & $H x_{2}$ & $H x_{3}$ \\
\cline { 2 - 3 }$H \widehat{e}_{2}$ & $H x_{2} \widehat{e}_{2}$ & $H x_{3} \widehat{e}_{2}$ \\
\hline
\end{tabular}

The partition on the left corresponds to the induced action on $H$ which cannot be primitive by Corollary 33 . The action on the right is primitive as it corresponds to the action we constructed in Theorem 40 .

\subsubsection{Case $2-|\Omega e|=1$}

Assume that $M$ has a primitive action on $\Omega$. If $|\Omega e|=1$, then $H=G_{e}=\{e\}$; Moreover, condition $|\Omega| \geq 3$ implies $n \geq 3$. By Corollary 21, there is only one action of $M$ on $\Omega$, up to equivalence.

$$
e M=\begin{array}{|l|l|l|l|}
\hline e & e_{2} & \cdots & e_{n} \\
\hline
\end{array}
$$

Moreover, $M \cong \bar{M}$ and $M$ is isomorphic to a submonoid of $\mathrm{T}_{\mathrm{n}}$, the transformation monoid on $n$ elements. Applying Corollary 8, $e M=\mathrm{I}(M)$ and $\mathrm{I}(M)$ is the set of constant mappings $\varsigma_{j}$, for $j=1, \cdots, n$.

Due to the nature of this case, we have not found a complete characterisation of such monoids. Of course, the natural action of the full transformation monoid $T_{n}$ on the set $\mathrm{n}=\{1, \cdots, n\}$ is always primitive. However, we have found many submonoids of $\mathrm{T}_{\mathrm{n}}$ having also primitive actions on $\mathrm{n}$. A first attempt in GAP4 [12] for $n=3$, the smallest possible case, returned several submonoids of $\mathrm{T}_{3}$ with primitive, faithful, and transitive actions on 3. At least we found monoids of size $6,8,9,10,11,12,13,16,17,22,23,24$, and 27 with 
this property. The description of this kind of monoids seems intractable with the current techniques, and therefore it remains as an open question.

However, there still quite a bit of structure for these type of monoids based on the study of the possible ranks of the elements of $M$ as a submonoid of $\mathrm{T}_{\mathrm{n}}$. We hope that our next results could help in future characterisations.

\section{On the study of ranks}

Let $M$ be a monoid having a primitive, transitive and faithful action on a set with $n$ elements. Suppose $G_{e}$ is trivial for some idempotent $e$ in I $(M)$. Assume towards a contradiction that $M=\mathrm{I}(M)^{1}=\mathrm{I}(M) \cup\{1\}$, then for $j \neq j^{\prime}$, the relation

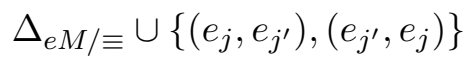

is a non-trivial congruence on $e M / \equiv$. As the action is primitive, this congruence must be equal to the total relation $\nabla_{e M / \equiv}$, which implies that $e M$ has only two idempotents, contradicting the fact that $n \geq 3$. Hence, there exists an element $m \in M$, different from the identity 1 , with $\operatorname{rank}(m)>1$ (as a mapping of $\mathbf{T}_{n}$ ).

This argument, based on the rank of the elements on $M$, can be extended to more general situations. In order to obtain refinements of the above argument, we will introduce further concepts. Denote the set of all two-sided ideals in $M$ by $\mathcal{I}(M)$. It is clear that $(\mathcal{I}(M), \subseteq)$ is a finite partially ordered set by inclusion. It has a lower bound given by $\mathrm{I}(M)$, whereas $M$ itself is an upper bound in $\mathcal{I}(M)$. Moreover, it is closed under finite intersections. The product of two ideals $I, J \in \mathcal{I}(M)$ given by $I J=\{i j \mid i \in I, j \in J\}$ is an ideal. It satisfies $\emptyset \neq I J \subseteq I \cap J$. Let $\Phi$ be an arbitrary $M$-set and let $I$ be a two-sided ideal of $M$. We define the $I$-center of $\Phi$, denoted by $\mathrm{Z}_{I}(\Phi)$ to be a new relation in $\Phi$ defined as follows

$$
(\alpha, \beta) \in \mathrm{Z}_{I}(\Phi) \quad \text { if and only if } \forall m, n \in I(\alpha m=\alpha n \Leftrightarrow \beta m=\beta n)
$$

Proposition $42([4])$. The center $\mathrm{Z}_{I}(\Phi)$ of an $M$-set $\Phi$ is a congruence in $\Phi$ for every ideal I in $M$.

As expected, the center congruences reverse inclusions.

Proposition 43. Let $I, J$ be two ideals in $M$ with $I \subseteq J$, then $Z_{J}(\Phi) \subseteq Z_{I}(\Phi)$.

If the transitive and faithful action of $M$ on $\Omega$ is primitive, then either $\mathrm{Z}_{I}(\Omega)=\Delta_{\Omega}$ or $\mathrm{Z}_{I}(\Omega)=\nabla_{\Omega}$, for every ideal $I \in \mathcal{I}(M)$.

Proposition 44. If $|\Omega e|=1$ for some idempotent $e \in \mathrm{I}(M)$, then $\mathrm{Z}_{\mathrm{I}(M)}(\Omega)=\nabla_{\Omega}$.

Proof. Applying Corollary 8, $e M=\mathrm{I}(M)$ and $\mathrm{I}(M)$ is the set of constant mappings on $\varsigma_{j}$ on $\Omega$, for $j=1, \cdots, n$. It follows that all elements in $\Omega$ are related in $\mathrm{Z}_{\mathrm{I}(M)}(\Omega)$. Therefore $\mathrm{Z}_{\mathrm{I}(M)}(\Omega)=\nabla_{\Omega}$.

In fact, last condition characterises $\mathrm{I}(M)$. It will follow from the following Lemma.

Lemma 45. Let $M$ be a monoid having a primitive, transitive and faithful action on $\Omega$. Assume that $|\Omega e|=1$ for some idempotent $e \in \mathrm{I}(M)$. For an ideal $I$ of $M$, the following statements are equivalent:

i) I contains an element $m$ with $\operatorname{rank}(m) \geq 2$; 
ii) $\mathrm{Z}_{I}(\Omega)=\Delta_{\Omega}$.

Proof. Assume that $\mathrm{Z}_{I}(\Omega)=\nabla_{\Omega}$. Then for all $\alpha, \beta \in \Omega$ and all $p, q \in I$ the following equation is satisfied

$$
\alpha p=\alpha q \quad \text { if and only if } \quad \beta p=\beta q .
$$

Fix some $\alpha \in \Omega$. Recall that $m$ and $\varsigma_{\alpha m}$ are elements in $I$, and the equation $\alpha m=\alpha\left(\varsigma_{\alpha m}\right)$ holds, therefore $\beta m=\beta \varsigma_{\alpha m}$ for all $\beta \in \Omega$. As the action is faithful, we conclude $m=\varsigma_{\alpha m}$, contradicing the fact that $\operatorname{rank}(m) \geq 2$. Hence, $\mathrm{Z}_{I}(\Omega)=\Delta_{\Omega}$. On the other hand, assume $I$ does not contain any element $m$ with $\operatorname{rank}(m) \geq 2$. Then $I=\mathrm{I}(M)$, which contradicts the assumption $\mathrm{Z}_{I}(\Omega)=\Delta_{\Omega}$.

Whereas the minimum ideal $\mathrm{I}(M)$ is mapped to a maximal center (in fact, it is mapped to the greatest possible congruence), all the other ideals are mapped to the minimal center (in fact, they are mapped to the smallest possible congruence). In an arbitrary $M$-set different gradings could occur, but primitive $M$-sets are extreme cases. Consider the set of ideals

$$
\mathbb{D}=\left\{I \in \mathcal{I}(M) \mid \mathrm{Z}_{I}(\Omega)=\Delta_{\Omega}\right\} .
$$

As expected, $\mathbb{D}$ stands for diagonal. This set has some interesting properties.

Proposition 46. Let $M$ be a monoid having a primitive, transitive and faithful action on $\Omega$. Assume that $|\Omega e|=1$ for some idempotent $e \in \mathrm{I}(M)$. Then $\mathbb{D}$ is a filter in $(\mathcal{I}(M), \subseteq)$.

Proof. Clearly $M$ is an element in $\mathbb{D}$, thus $\mathbb{D}$ is not empty. If $I \in \mathbb{D}$ and $I \subseteq J$, Proposition 43 implies that $\mathrm{Z}_{J}(\Omega) \subseteq \mathrm{Z}_{I}(\Omega)=\Delta_{\Omega}$, thus $\mathrm{Z}_{J}(\Omega)=\Delta_{\Omega}$. Now, let $I, J$ be two ideals in $\mathbb{D}$, then for all $\alpha, \beta \in \Omega$ with $\alpha \neq \beta$, the pair $(\alpha, \beta) \notin \mathrm{Z}_{I}(\Omega)=\Delta_{\Omega}$. Therefore, one can assume without loss of generality that there exist elements $m, n \in I$ with

(a) $\alpha m=\alpha n$ and $\beta m \neq \beta n$

again, as $\beta m \neq \beta n$ and $\mathrm{Z}_{J}(\Omega)=\Delta_{\Omega}$, one can assume without loss of generality that there exists elements $p, q \in J$ with

(b) $\beta m p=\beta m q$ and $\beta n p \neq \beta n q$

Assume that $\mathrm{Z}_{I J}(\Omega)=\nabla_{\Omega}$, then the elements $\alpha, \beta$ in $\Omega$ considered above satisfy that for all $r, s \in I J, \alpha r=\alpha s$ if and only if $\beta r=\beta s$. The following double implications hold:

$$
\begin{array}{rlrl}
\beta m p=\beta m q & \Leftrightarrow & \alpha m p=\alpha m q & \left(\mathrm{Z}_{I J}(\Omega)=\nabla_{\Omega}, m p, m q \in I J\right) \\
& \Leftrightarrow \quad \alpha n p=\alpha n q & (\mathrm{By}(\mathrm{a}) \alpha n=\alpha m) \\
& \Leftrightarrow \quad \beta n p=\beta n q & \left(\mathrm{Z}_{I J}(\Omega)=\nabla_{\Omega}, n p, n q \in I J\right) .
\end{array}
$$

This contradicts (b). It follows that $\mathrm{Z}_{I J}(\Omega)=\Delta_{\Omega}$. Since $I J$ is always included in $I \cap J$, it follows that $Z_{I \cap J}(\Omega)=\Delta_{\Omega}$.

Corollary 47. If $M$ has a primitive, faithful and transitive action on $\Omega$ with $|\Omega e|=1$ for some idempotent $e \in \mathrm{I}(M)$, and $I$ is an ideal in $M$ containing an element $m$ with $\operatorname{rank}(m) \geq 2$, then for every non-zero natural number $k \in \mathbb{N}$, the ideal $I^{k}$ contains an element $p$ with $\operatorname{rank}(p) \geq 2$.

We hope that the above corollary can be used to further understand primitive, faithful and transitive actions of monoids $M$ whose maximal subgroups at the idempotents of $\mathrm{I}(M)$ are trivial. 


\section{Acknowledgements}

The first author has been supported by the grant MTM2014-54707-C3-1-P from the Ministerio de Economía y Competitividad (Spanish Government), the grant PROMETEO/2017/057 from the Generalitat, Valencian Community, Spain, and the grant 11271085 from the $N a$ tional Natural Science Foundation of China. The second author has been supported by the grant MTM2014-54707-C3-1-P from the Ministerio de Economía y Competitividad (Spanish Government). The third author has been supported by the grant MTM2010-19938-C03-03 from the Ministerio de Ciencia e Innovación (Spanish Government).

We are very grateful to the referee for the careful and meticulous reading of the paper as well as for the very helpful comments.

\section{References}

[1] J. Almeida, S. Margolis, B. Steinberg, and M. Volkov. Representation theory of finite semigroups, semigroup radicals and formal language theory. Trans. Amer. Math. Soc., 361:1429-1461, 2009.

[2] M. Arbib, K. Krohn, and J. Rhodes. Algebraic theory of machines, languages, and semigroups. Academic Press, 1968.

[3] A. Ballester-Bolinches and L. M. Ezquerro. Classes of Finite Groups, volume 584 of Mathematics and Its Applications. Springer, Dordrecht, 2006.

[4] S. Burris and H. Sankappanavar. A course in universal algebra. Graduate texts in mathematics. Springer-Verlag, 1981.

[5] P. Cameron. Permutation Groups, volume 45 of London Mathematical Society Student Texts. Cambridge University Press, 1999.

[6] A. H. Clifford and G. B. Preston. The algebraic theory of semigroups, vol. I. Amer. Math. Soc., Providence, RI, USA, 1961.

[7] A. H. Clifford and G. B. Preston. The algebraic theory of semigroups, vol. II. Amer. Math. Soc., Providence, RI, USA, 1967.

[8] J. D. Dixon and B. Mortimer. Permutation Groups. Graduate Texts in Mathematics. Springer, New York-Berlin-Heidelberg, 1996.

[9] K. Doerk and T. Hawkes. Finite soluble groups, volume 4 of De Gruyter Expositions in Mathematics. Walter de Gruyter \& Co., Berlin, 1992.

[10] O. Ganyushkin, V. Mazorchuck, and B. Steinberg. On the irreducible representations of a finite semigroup. Proc. Amer. Math. Soc., 137:3585-3592, 2009.

[11] O. Ganyushkin and V. Mazorchuk. Classical Finite Transformation Semigroups: An Introduction. Algebra and Applications. Springer, 2008.

[12] The GAP Group. GAP - Groups, Algorithms, and Programming, Version 4.7.8, 2015. 
[13] J. Green, K. Erdmann, and M. Schocker. Polynomial Representations of $G L_{n}$ : With an Appendix on Schensted Correspondence and Littelmann Paths. Lecture Notes in Mathematics. Springer, 2007.

[14] J. M. Howie. Fundamentals of Semigroup Theory. LMS monographs. Clarendon Press, 1995.

[15] A. Kerber. Applied Finite Group Actions. Algorithms and Combinatorics. Springer, 1991.

[16] M. Kilp, U. Knauer, and A. Mikhalev. Monoids, Acts, and Categories: With Applications to Wreath Products and Graphs. A Handbook for Students and Researchers. De Gruyter Expositions in Mathematics. De Gruyter, Berlin, Boston, 2000.

[17] S. A. Linton, G. Pfeiffer, E. F. Robertson, and N. Ruškuc. Groups and actions in transformation semigroups. Math. Z., 228(3):435-450, 1998.

[18] S. A. Linton, G. Pfeiffer, E. F. Robertson, and N. Ruškuc. Computing transformation semigroups. J. Symbolic Comput., 33(2):145-162, 2002.

[19] J.-E. Pin. Le problème de la synchronisation et la conjecture de Černý. Thèse de 3ème cycle, Université Paris VI, 1978.

[20] J.-E. Pin. On two combinatorial problems arising from automata theory. Annals of Discrete Mathematics, 17:535-548, 1983.

[21] I. S. Ponizovskiur. Transitive representations by transformations of semigroups of a certain class. Sibirsk. Mat. Zh., 30(5):896-903, 1964. Russian. Translation in Nineteen papers on algebraic semigroup theory. Amer. Math. Soc. Transl., 139, no. 2 (1988), pages 85-92.

[22] D. Rees. On semi-groups. Math. Proc. Cambridge Philos. Soc., 36:387-400, 1940.

[23] J. Rhodes and B. Steinberg. The q-theory of finite semigroups. Springer Monographs in Mathematics. Springer, New York, 2009.

[24] I. K. Rystsov. On the length of reset words for automata with simple idempotents. Kibernet. Sistem. Anal., 3:32-39, 2000.

[25] B. Steinberg. A Theory of Transformation Monoids: Combinatorics and Representation Theory. Electron. J. Combin, 17(1):Research Paper 164, 56 pp., 2010.

[26] J. Černý. A remark on homogeneous experiments with finite automata. Mat.-Fyz. Časopis Sloven. Akad. Vied, 14:208-216, 1964. 